\title{
Multi-plane time-resolved Particle Image Velocimetry (PIV) flow field measurements in an optical Spark-Ignition Direct-Injection (SIDI) engine for Large-Eddy Simulation (LES) model validations
}

\author{
Fengnian Zhao ${ }^{1}$, Mengqi Liu ${ }^{1}$, Penghui Ge ${ }^{1}$, David L.S. Hung ${ }^{1,2,}{ }^{*}$, Xuesong Li $^{2}$, Min Xu $^{2}$, Xiaofeng Yang ${ }^{3}$, \\ and Cherian Idicheria ${ }^{3}$ \\ ${ }^{1}$ University of Michigan-Shanghai Jiao Tong University Joint Institute, Shanghai Jiao Tong University, 800 Dongchuan Rd, \\ 200240, Shanghai, China \\ ${ }^{2}$ Institute of Automotive Engineering, School of Mechanical Engineering, Shanghai Jiao Tong University, 800 Dongchuan Rd, \\ 200240, Shanghai, China \\ ${ }^{3}$ GM Global Research and Development, 800 N Glenwood Ave., Pontiac, MI 48340-2925, USA
}

Received: 15 December 2018 / Accepted: 8 April 2019

\begin{abstract}
In-cylinder flow characteristics play a significant role in the fuel-air mixing process of Spark-Ignition Direct-Injection (SIDI) engines. Typically, planar Particle Image Velocimetry (PIV) is used to measure a representative velocity field sectioning through the center plane of the engine cylinder. However, a single flow field offers very limited perspective regarding the Three-Dimensional nature of the flow fields. Since the in-cylinder flow is stochastically complex, large datasets of flow field measurements along multiple planes are needed to provide a complete panoramic understanding of the flow dynamics. In this study, a high-speed PIV is applied to measure the crank-angle resolved flow fields inside a single-cylinder four-valve optical SIDI engine. Five flow fields along different tumble planes are captured. These five planes include two orthogonal planes cutting through the spark plug tip, two parallel planes sectioning through middle point of the intake and exhaust valves, and one plane through the centers of two intake valves. In addition, numerical computations are carried out with Large-Eddy Simulation (LES) model in CONVERGE. With the guidance from multi-plane PIV measurements, a novel validation approach is proposed in this study. The quantitative analysis and comparison between LES simulations and PIV experiments are divided in terms of global and local comparison indices. The global comparison indices provide a quantitative single value to quickly check the overall similarity of velocity directions and magnitudes between PIV and LES results of a specific individual plane. The local comparison indices further evaluate the similarity between the flow fields of LES and PIV point by point to identify any dissimilar regions and vortex features, which are likely to indicate the complex flow structures at low-speed regions. In summary, not only can the combined data analysis approach provide a reliable way for LES model validations, it can also reveal the physical quantifications of the complex in-cylinder flow characteristics.
\end{abstract}

\section{Introduction}

Spark-Ignition Direct-Injection (SIDI) engines have shown potential benefits of lower fuel consumption, better power output, and reduced emissions over port fuel injection engines. Specifically, the flow field characteristics inside a SIDI engine cylinder play a critical role in governing the fuel mixture formation and engine combustion. However, the nature of in-cylinder flow fields is highly complex and cyclic which have not yet been thoroughly investigated. To elucidate these characteristics, Large-Eddy Simulation (LES) has been applied to simulate the in-cylinder flow fields

\footnotetext{
* Corresponding author: dhung@sjtu.edu.cn
}

and quantify the cyclic variations of engine flows under motoring or firing operations [1-5]. The high temporal evolution of the flow fields predicted by LES results improves the understanding of critical in-cylinder process with time-resolved information.

In order to construct reliable LES models, the simulation results of in-cylinder flow fields with high temporal and spatial resolutions are commonly validated with highspeed Particle Image Velocimetry (PIV) data [4-7]. In most research, planar PIV is used to measure the flow fields to validate the LES model by focusing only on the velocity comparison on a specific plane [8, 9] or a targeted spatial zone or location near the intake valve $[5,10]$. When comparing the velocity results between PIV and LES, the 
Relevance Index (RI) is commonly used. It evaluates the structure similarity by calculating the projection of one velocity field onto another one. Since RI is useful to quantify the degrees of similarity of two velocity fields, many researchers applied RI to check the equivalence of PIV and LES flow fields $[11,12]$. Beside the comparison of velocity contours, some research has been focused on the tumble structure of flow fields [13]. With the improvements of PIV in recent years, flow field measurements of more than one plane or 3D PIV measurements have been conducted, allowing more flow structures to be thoroughly investigated. For instance, Buhl et al. [12] utilized the "Flying PIV" to validate the flow structures near the piston surface. Nicollet et al. [8], Bode et al. [14], and Baum et al. [15] used a quasi-simultaneous time-resolved approach to correlate PIV results of two adjacent planes and the Tomo-PIV to visualize the $3 \mathrm{D}$ flow structures within millimetric thickness. However, 3D PIV technique is confirmed to a finite, "thin-volume" measurement so that it is hard to reveal the flow structure of the whole cylinder domain. For "Flying PIV" and scanning PIV, these measurement techniques are quite innovative, but the selections of the measured plane are not flexible, which are mostly constrained by the experimental setup and hardware configuration of the engine. In most other situations, multi-plane PIV are also conducted to provide a panoramic understanding of the flow dynamics $[16,17]$. However, in order to capture flow data of more planes, the multi-plane PIV data is recorded separately for every plane from different engine cycles.

In this work, the PIV flow field measurements of five tumble planes are captured. Figure 1 shows the positions of all five tumble planes from the top view of the cylinder. These five focal planes include all crucial regions inside the cylinder, which are two orthogonal planes through the spark plug tip (BB and $\mathrm{EE}$ ), two planes sectioning through the intake valve and exhaust valve (CC and $\mathrm{AA})$, and one plane along two intake ports (DD).

There are two objectives of this experimental-centric study: the first objective is to improve the understanding of flow structure regarding its spatial and temporal development inside the whole cylinder. The second objective is to provide guidance for the validation of LES model using multi-plane crank angle-resolved PIV measured flow field data. To compare the PIV and LES results of each specific plane, different maps of local comparison indices are constructed in addition to the single value of the Global Structure Index (GSI) (also known as RI) and magnitude index. The benefit of using local comparison index over a single value comparison is that it shows the specific dissimilar regions between two sets of results. After these dissimilar regions are identified, both velocity and flow feature comparisons are conducted to quantify the correlations between PIV and LES. In the following sections, the experimental setup is first introduced, followed by the numerical approach section to provide details of LES model. Next, the multi-plane PIV results are discussed to reveal flow characteristics inside the cylinder. In the LES validation section, a multi-plane validation based on hierarchical validation method combining velocity profile and flow features comparisons is conducted. Finally, conclusions are drawn to

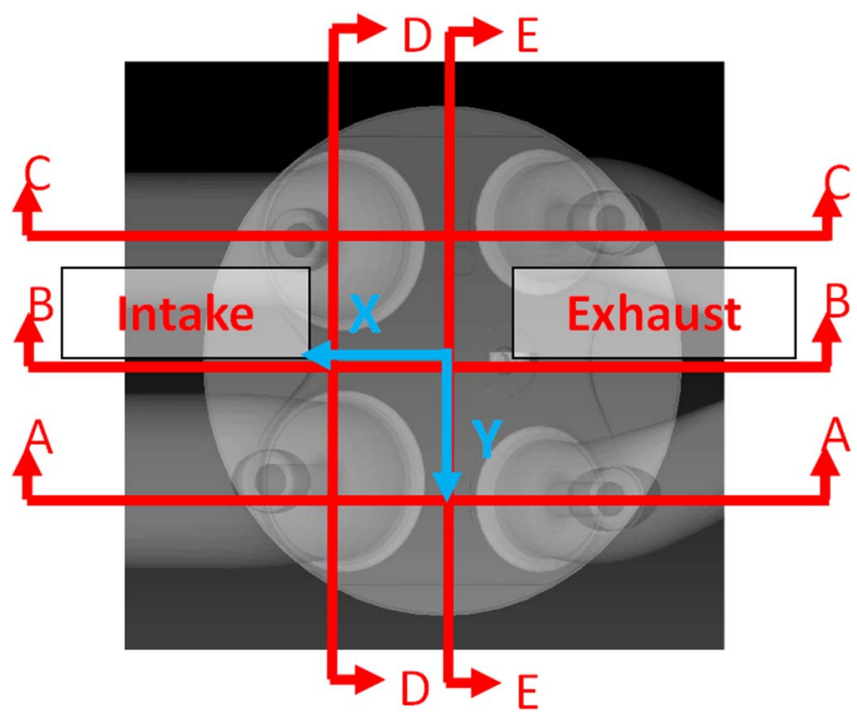

Fig. 1. Tumble plane positions of PIV measurement.

highlight the benefits of the multi-plane comparison methodology in this paper.

\section{Experimental setup}

As depicted in Figure 2, the optical engine in this study is based on a production $2.0 \mathrm{~L}$ four-valve SIDI engine configuration. The key components are the optical piston insert and optical liner, and both are made of high-quality quartz material. The optical piston insert provides an optical access for the laser sheet to illuminate the target measurement plane in the cylinder, and the optical liner allows the high-speed camera to record the flow fields. Figure 3 shows the schematic of PIV setup. Silicon oil particles of about $2 \mu \mathrm{m}$ average diameter were generated by a TSI six-jet atomizer. These seeding particles were mixed with the fresh air in the intake port prior to entering the cylinder. A double-pulsed Nd:YLF laser with $527 \mathrm{~nm}$ wavelength (LaVision DM30$527-\mathrm{DH})$ was used to illuminate the silicon oil droplets through a $45^{\circ}$ mirror beneath the optical piston. The highspeed camera (Phantom V7.3) was used to record pairs of image at every five CADs. The images were processed using the commercial code of LaVision Davis 8.4. The vector choice of each vector in the flow field is used to check the quality and validity of PIV results. The first-choice vectors are determined directly by the cross-correlations of the seeding particles in the images without post-processing. The rest spurious vectors will be interpolated using neighboring non-zero vectors. In this study, the percentage of first-choice vectors of PIV data was over $96 \%$, which means that the interpolated vectors are less than $4 \%$. Table 1 shows the detailed parameters of the PIV setup.

In this study, the PIV results were recorded under a motoring engine speed of $1300 \mathrm{rpm}$ with an intake manifold pressure of $40 \mathrm{kPa}$. The velocity fields were captured at every five $\mathrm{CAD}$ from $-300 \mathrm{CAD}$ to $-60 \mathrm{CAD}$ for 

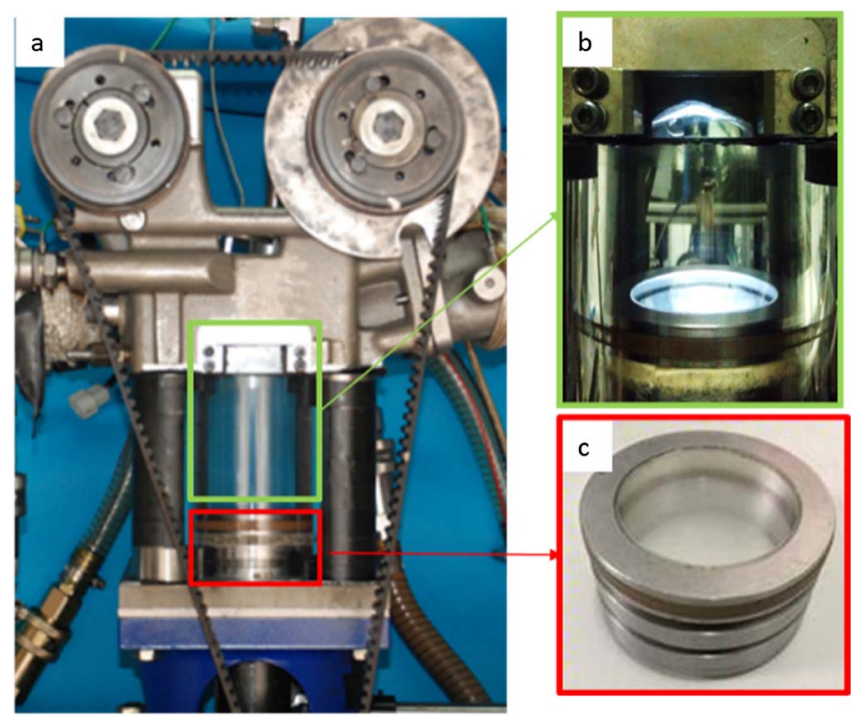

Fig. 2. (a) Optical engine; (b) Quartz liner; (c) Optical piston with quartz insert.

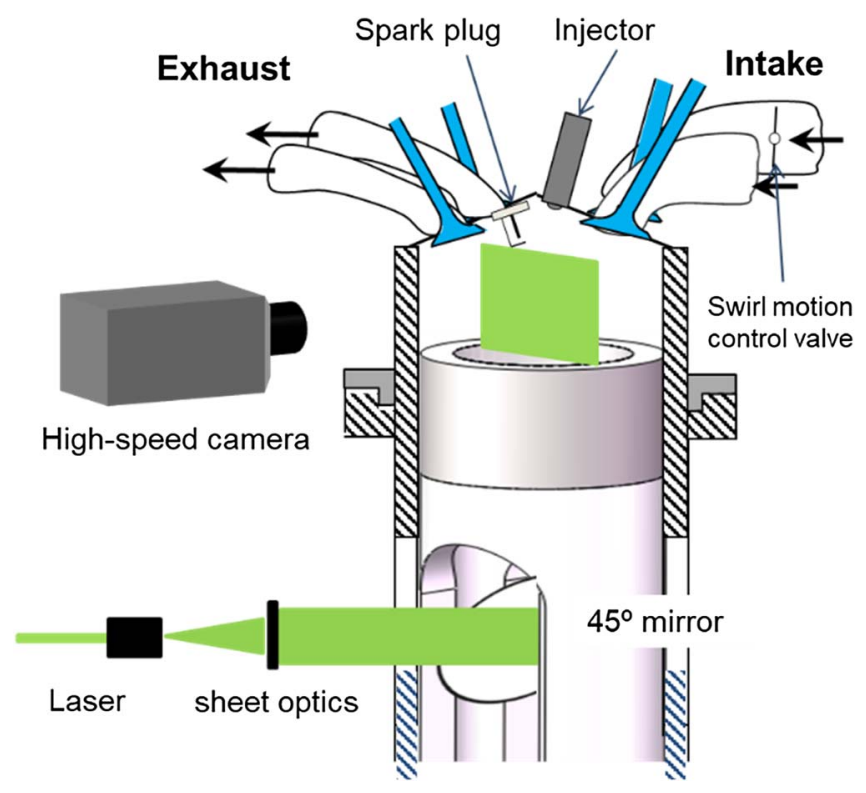

Fig. 3. Schematic of PIV setup.

100 consecutive cycles. The detailed engine parameters and operating conditions are listed in Table 2.

\section{Numerical approach}

The in-cylinder flow field exhibits complex mechanism with convoluted Three-Dimensional flow features. Due to the limitations of experimental approach, exploring the mechanism of flow field requires the reliable 3-D simulation model. LES has become one of the mostly accepted methods in modeling the in-cylinder flow field owing to its advantages of high accuracy and efficiency. To ensure the accuracy of
LES, the initial and boundary conditions must be correctly stated. However, typical data provided only by the mean values of experiments cannot provide adequate information for LES simulations. Therefore, in this modeling, the 1D-3D simulation approach is adopted. Precisely calibrated one-dimensional flow model is used to provide accurate boundary and initial conditions for LES simulations $[4,7]$. In our study, this 1D model method is achieved using GT-Power v7.4. The results from the 1-D model are validated using specific engine measurements. The 1-D results from GT-Power are used as the appropriate ICs and BCs for 3D LES study of in-cylinder flow which was conducted with a commercial CFD code CONVERGE v2.1.

\subsection{1-D GT-Power model}

The schematic diagram of 1D GT-Power flow model is shown in Figure 4. This model includes modules for the optical engine, an atomizer for silicon oil, and the throttle valve and heater. The temperature and pressure of the intake side (intake plenum, runners and ports) were carefully calibrated with the experimental data. All dimensions of the related components in the system model were verified from the actual hardware. An atomizer was also added to the GT-Power model to include the influence of tracing particles on PIV measurements. A throttle valve was used to maintain the absolute intake pressure at $40 \mathrm{kPa}$. A heater model was added for the intake air temperature control, but it was not switched on for this study.

The valve timing and profile of the running engine are shown in Figure 5a. The intake valves opened at -384 CAD and closed at -100 CAD, and $117 \mathrm{CAD}$ and 401 CAD for the exhaust valves, respectively. PIV measurements were conducted during the crank angle range of $240 \mathrm{CAD}$ from early intake stroke to late compression stroke $(-300 \mathrm{CAD}$ to $-60 \mathrm{CAD})$. Experimental data of crank angle based pressure obtained from intake port, exhaust port, and cylinder were used to validate this 1-D model as shown in Figures 5b-5d. It is reasonable to observe that the average intake port pressure and exhaust port pressure were around $40 \mathrm{kPa}$ and $100 \mathrm{kPa}$, respectively, because they were consistent with the intake and exhaust boundary conditions. The pressure fluctuations of intake and exhaust ports increased when the corresponding valves opened. The in-cylinder pressure captured by the experiments matched quite well with the GT-Power results. An error percentage is used to quantify the difference between experiments and 1-D simulations, which is defined as:

$$
\text { Error percentage }(\%)=\left|\frac{\operatorname{Exp}_{\text {pressure }}-\mathrm{GT}_{\text {pressure }}}{\operatorname{Exp}_{\text {pressure }}}\right| \times 100 \% \text {, }
$$

where $\operatorname{Exp}_{\text {pressure }}$ is the pressure data from measurements, and $\mathrm{GT}_{\text {pressure }}$ represents the pressure data using the GT-Power model.

From the comparison results, the error percentage of intake pressure is below $2 \%$, while the exhaust and in-cylinder pressures are relatively larger, but mostly less than $5 \%$. One possible reason is that the in-cylinder and 
Table 1. PIV parameters.

\begin{tabular}{ll}
\hline Parameter & Value \\
\hline Particle image size & $3-4$ pixels/particle \\
Interrogation size and overlap & $32 \times 32$ pixels and $50 \%$ \\
Particle image density per interrogation window & $10-15$ particles \\
Laser sheet thickness & $1 \mathrm{~mm}$ \\
Time interval between two pulses & $20 \mu \mathrm{s}$ \\
Frame resolution & 1 frame $/ 5$ CAD \\
Spatial resolution & Planes AA and DD: $0.121 \mathrm{~mm} / \mathrm{pixel}$ \\
& Planes BB and EE: $0.133 \mathrm{~mm} / \mathrm{pixel}$ \\
\hline
\end{tabular}

Table 2. Engine and operating parameters.

\begin{tabular}{lc}
\hline Parameter & Value \\
\hline Engine speed & $1300 \mathrm{rpm}$ \\
Bore & $86 \mathrm{~mm}$ \\
Stroke & $94.6 \mathrm{~mm}$ \\
Clearance volume & $49.96 \mathrm{~cm}^{3}$ \\
Engine displacement & $549.51 \mathrm{~cm}^{3}$ \\
Compression ratio & $12: 1$ \\
Manifold absolute pressure & $40 \mathrm{kPa}$ (absolute) \\
\hline
\end{tabular}

exhaust pressure results are more affected by the piston movement, giving rise to larger error differences. However, all these error percentages are reasonable based on the validation results obtained from a previous work [16]. It demonstrates that the 1-D simulation results are reliable as the initial and boundary conditions for the LES study.

\subsection{Large-Eddy Simulation (LES) model}

LESs were conducted using a finite volume based CONVERGE code. The computational domain is shown in Figure 6, which includes two intake ports, cylinder, and two exhaust ports. Thirty-two consecutive motored cycles were computed in total but the results from first two cycles were discarded. Fine grid structures together with advanced and well-defined turbulence models were employed in our LES work to include the complex features of in-cylinder flow fields.

The mesh arrangement is also depicted in Figure 6, and the number of cell ranges from 505188 (Top Dead Center, TDC) to 1080994 (Bottom Dead Center, BDC). The finest mesh spacing of $0.25 \mathrm{~mm}$ was placed near the valve seat regions. The surface structure mesh size was the smallest in this region since the geometry had a critical impact on the airflow entering the cylinder. Relatively finer mesh $(0.5 \mathrm{~mm})$ was used in the cylinder domain. The coarsest mesh of $2 \mathrm{~mm}$ was employed inside the intake and exhaust ports. For near wall regions, Werner and Wengle model [18] was applied to handle the flow boundary layer. Meanwhile, a modified cut-cell Cartesian method was applied by CONVERGE in order to minimize the influence of grid topology on the simulation results [19]. Considering that the experiments and simulations were carried out under non-combustion conditions, the temperature boundary conditions for solid walls were set to a constant value of $318 \mathrm{~K}$. The intake air temperature of $295 \mathrm{~K}$ was calibrated using experimental data at each crank angle.

The one-equation eddy viscosity dynamic structure turbulence model [20] was selected for the LES study. Different from the method of turbulent viscosity concept defined by user in the traditional one-equation LES model, a dynamic approach was conducted to calculate the sub-grid stress tensor more precisely in dynamic structure model. According to previous research [16], it is capable of better capturing the complex flow with high velocity gradients compared to the common one-equation LES model. Flow with large velocity gradients will be generated since tumble motion and random turbulence have high probability of appearing in the flow field inside the cylinder, especially for the nearsurface regions. Hence, dynamic structural model is well suited for in-cylinder flow modeling.

\section{Experimental results}

LES validation typically starts by selecting a flow field of interest which is the most common representative plane inside the cylinder. However, flow data of single or dual planes is not sufficient to represent the whole flow characteristics inside the cylinder. For example, the flow field under two intake valves during intake stroke will have quite different features compared with the flow field under two exhaust valves. Therefore, multi-plane time-resolved PIV focusing on all crucial planes provides more complete perspective of flow dynamic for validating the LES model. Based on the cylinder head design of the optical engine in this study, five representative tumble planes are selected as shown in Figure 1. For the following analysis, the PIV results at three representative CADs are discussed first, which are - 270 CAD (intake stroke), $-180 \mathrm{CAD}$ (bottom dead center), and -90 CAD (compression stroke). This section can be divided into two 


\section{Atomizer for silicon oil}

\section{Optical engine}

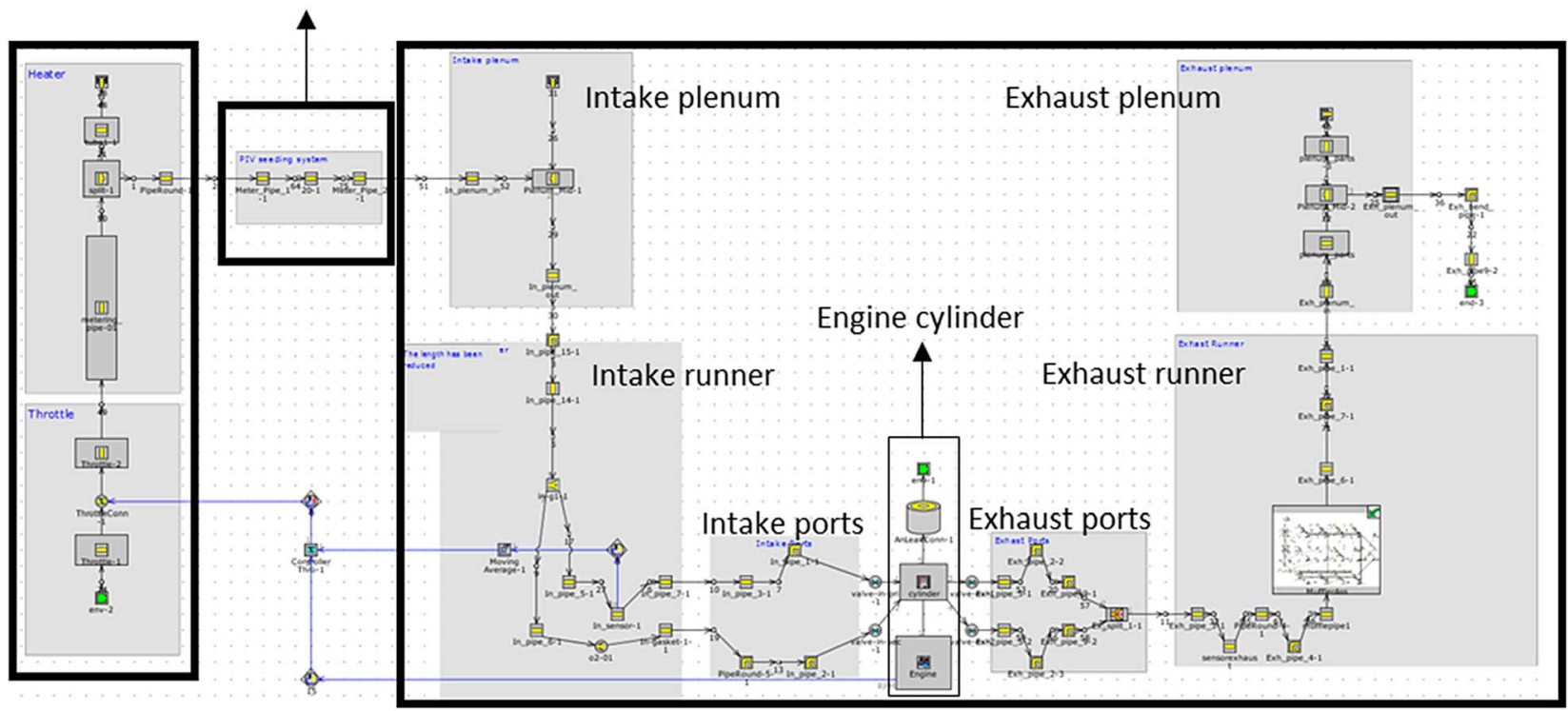

\section{Throttle valve \& heater}

Fig. 4. GT-Power model of optical engine.
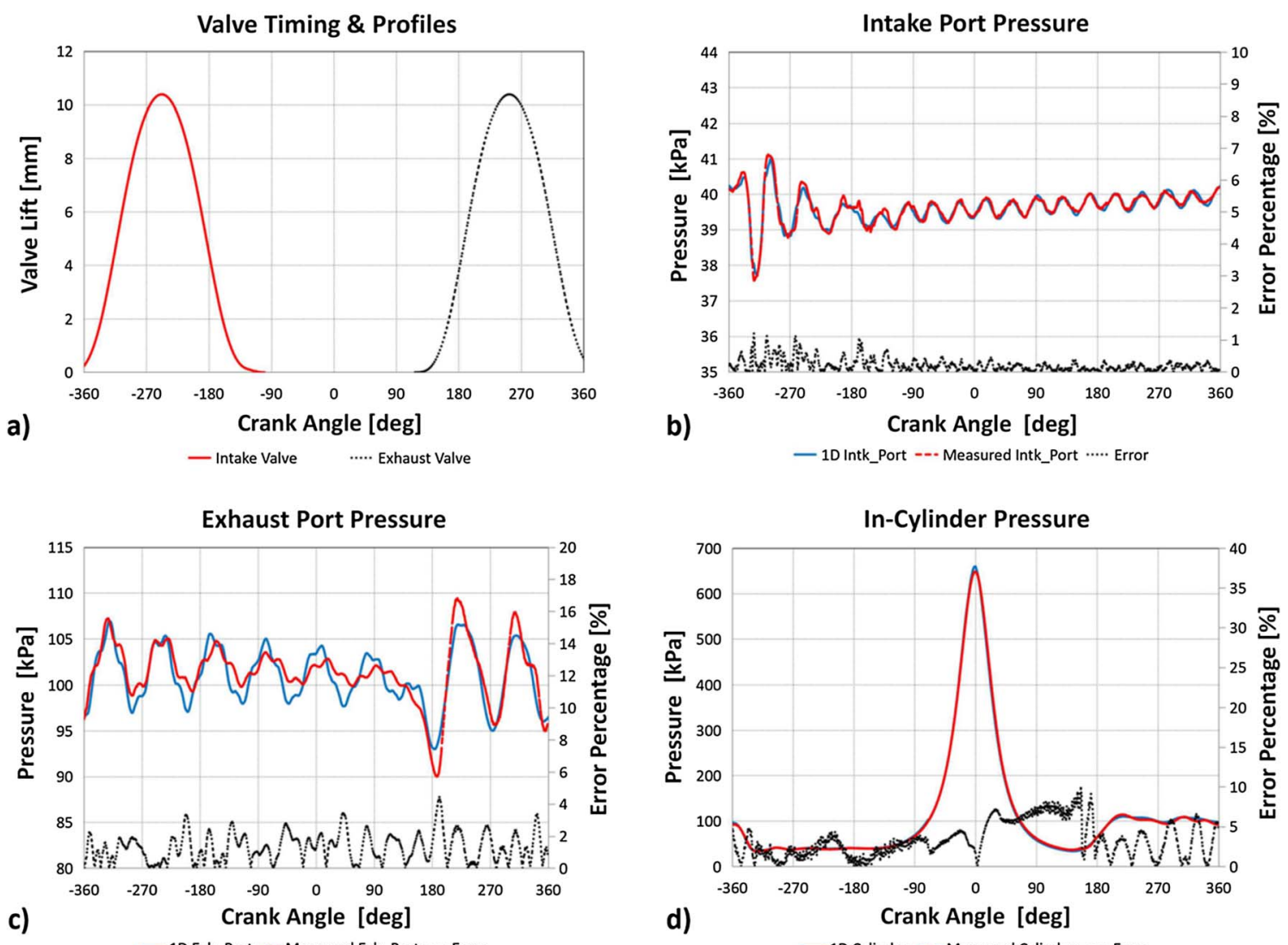

Fig. 5. Valve timing and quantitative comparison between measurements and GT-Power results. (a) Valve timing and profiles; (b) intake port pressure; (c) exhaust port pressure; (d) in-cylinder pressure. 


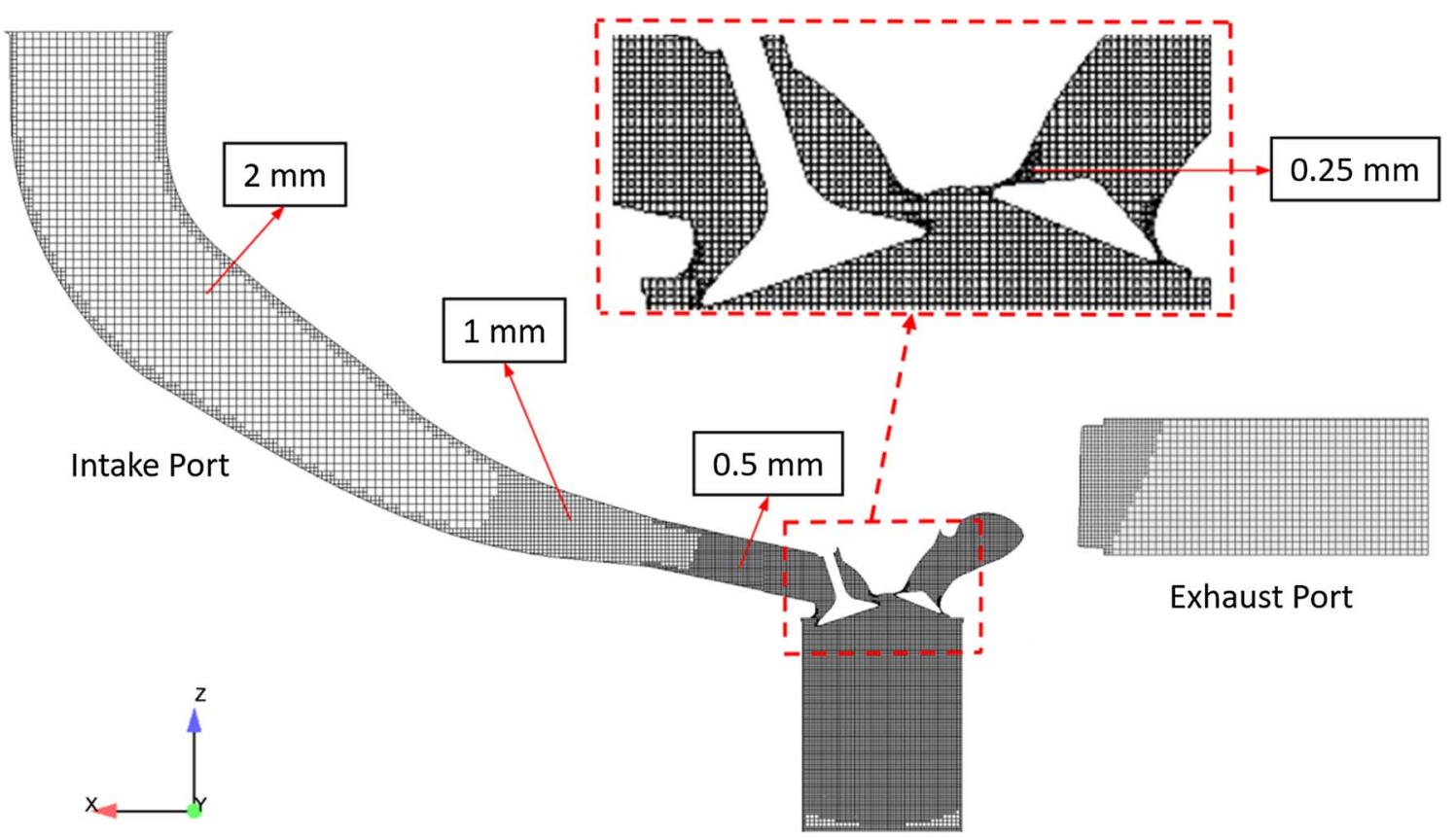

Fig. 6. Mesh arrangements used in the LES model.

parts. The first part is about PIV flow analysis of AA, CC, and $\mathrm{BB}$ planes, which are three parallel planes along the $X$-axis. The second part is PIV flow analysis of the other two planes (DD and EE) which are parallel to the $Y$-axis.

\subsection{PIV flow field measurements of AA, CC, and $B B$ planes}

Even though AA and $\mathrm{CC}$ planes are both sectioning through the intake valve and exhaust valve, the intake ports are designed with different angles of entry into the cylinder. Figure 7 shows the intake ports of $\mathrm{AA}$ and $\mathrm{CC}$ planes. The intake ports of AA and CC planes are labeled as Intake Port 1 (red) and Intake Port 2 (blue), and the angle between Intake Port 1 and $X$-axis is larger than that of Intake Port 2.

Figure 8 shows the PIV flow fields at $-270,-180$, and -90 CADs of AA and CC planes. At -270 CAD, the red arrows show a strong intake air entering the cylinder. Because of the inertia of intake air, the velocities at the right side (right dash line) of the flow fields are co-directional with relatively larger magnitude. The left side (left dash line) of flow fields of AA and CC planes also show the similar flow trends but the velocity magnitudes are much lower compared with the right side. As the incoming air continues to develop at the right side, the cylinder wall on the left side resists the inertia of intake air. Although the main flow patterns of AA and CC planes are similar (indicated by black arrows), the velocity magnitude on $\mathrm{CC}$ plane is relatively smaller than that along the AA plane. It is reasonable since the intake runner of AA plane provides a more vertical (tumble) intake flow direction into the cylinder. For the middle region (between the dash lines) of the flow field, a disordered low-speed flow exists for both planes because of

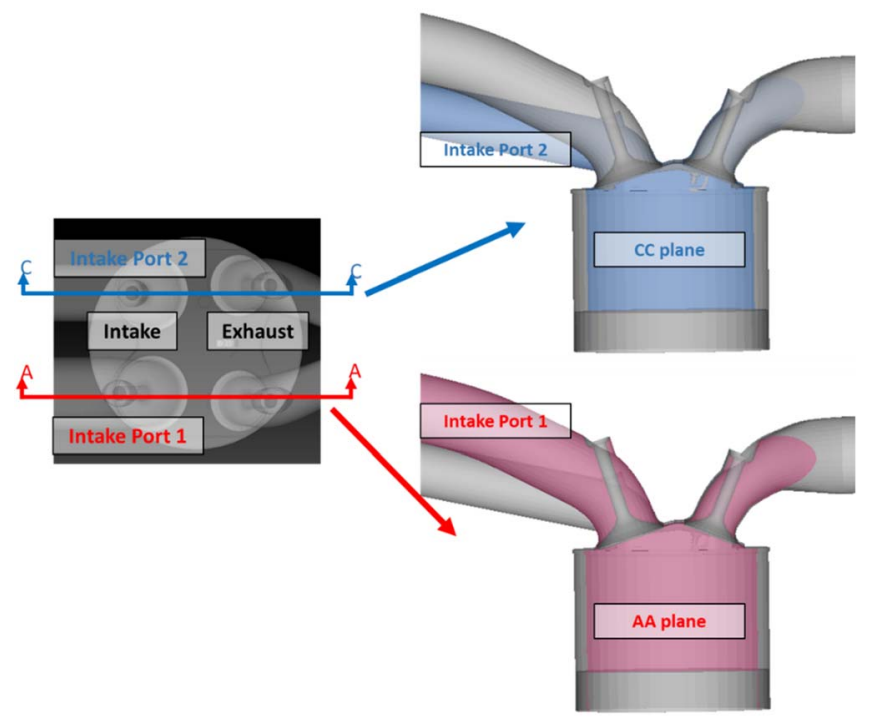

Fig. 7. Intake ports above AA plane (Intake Port 1) and CC plane (Intake Port 2).

the intake valve blockage. Another phenomenon of interest is the upward flow exists at the regions right behind the intake valves. The reason could be that the separation of the flow occurs after intake air passes over the edges of intake valves.

As the intake valve is gradually closing, the flow field becomes less complex compared with that during the intake stroke. The dominant clockwise tumble motions are formed for both AA and CC planes at -180 CAD. The velocities at AA plane are still relatively larger than that on $\mathrm{CC}$ plane. 


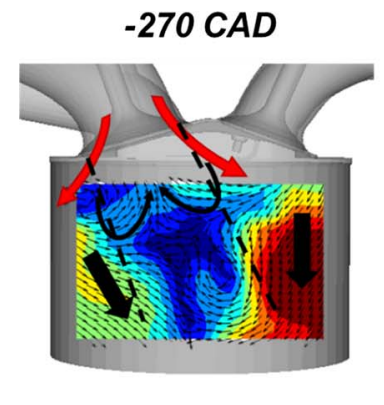

\section{AA plane}
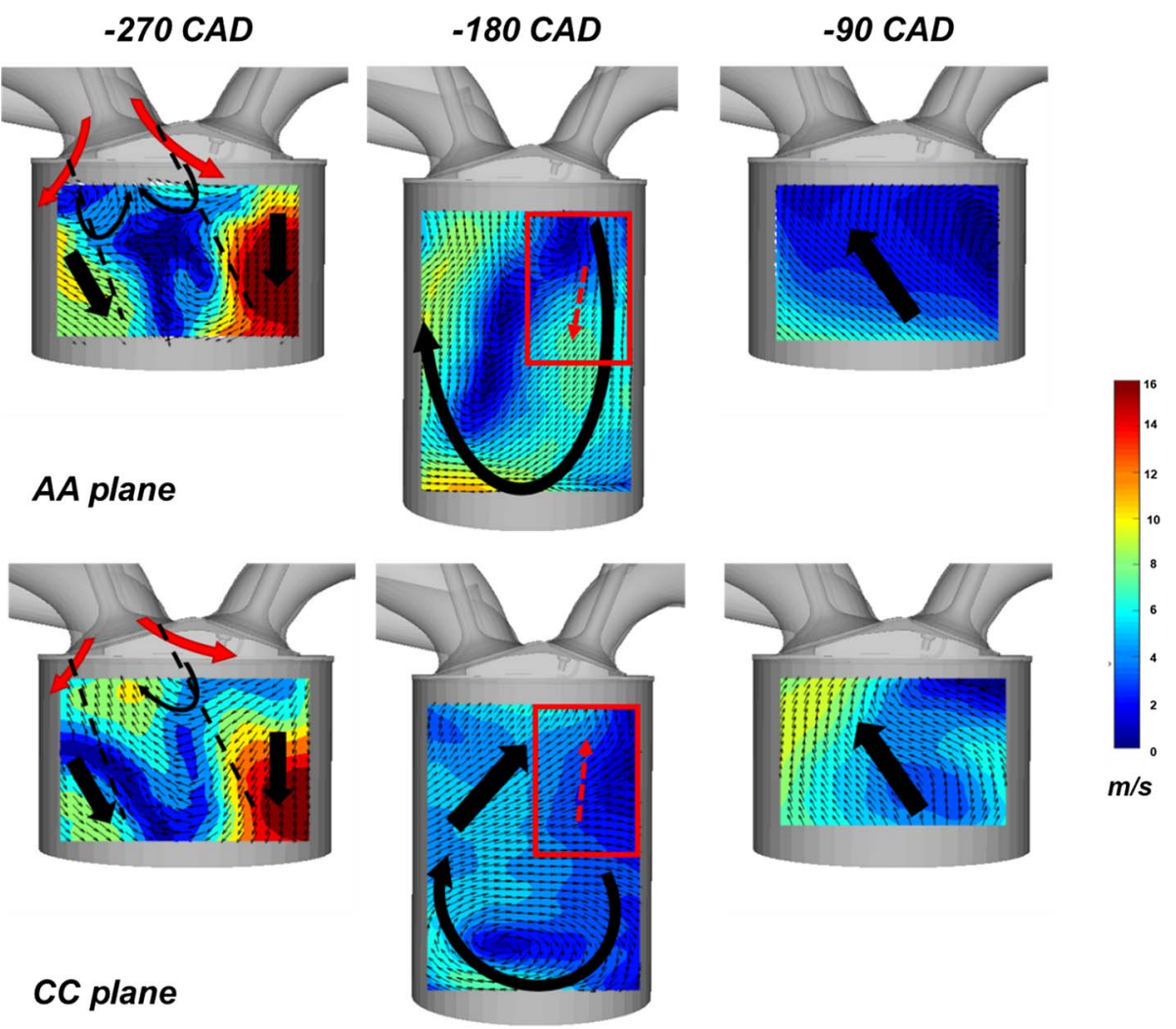

CC plane

Fig. 8. PIV results at $-270,-180$, and $-90 \mathrm{CAD}$ of AA and CC planes.
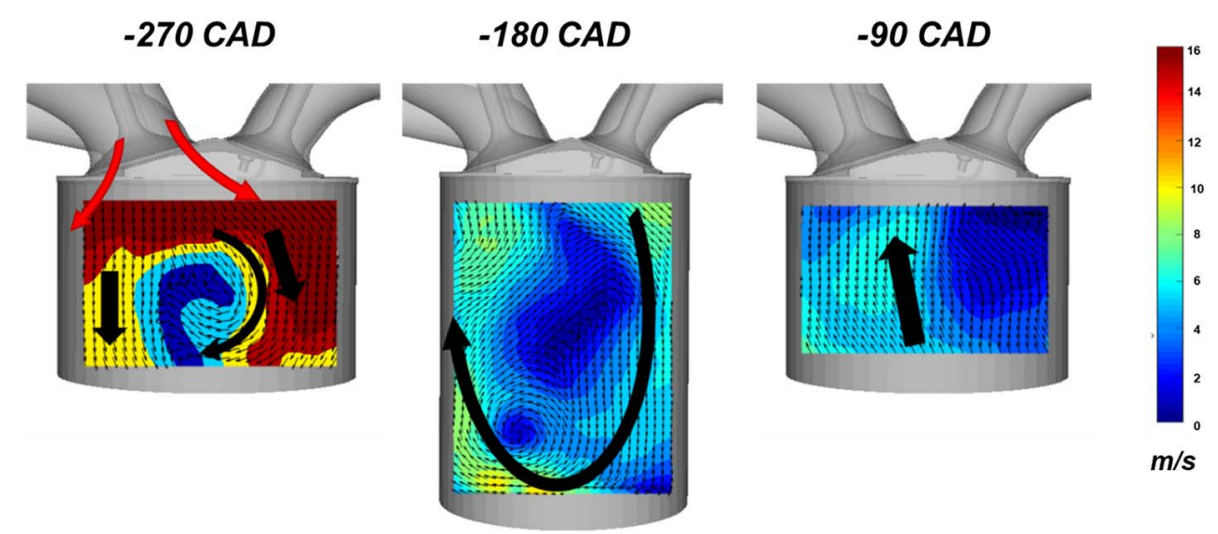

Fig. 9. PIV results at $-270,-180$, and $-90 \mathrm{CAD}$ of $\mathrm{BB}$ plane.

Another obvious difference is at the top right region (indicated by the red boxes) of the flow field. The velocity directions of CC plane are upward, which shows that the tumble motion on CC plane is not as strong as that along AA plane. During compression stroke, both flow fields turn into a simpler unidirectional flow pattern. The flow directions of both planes point upwards because of the upward movement of piston.

Figure 9 shows the PIV results of BB plane at -270 , -180 , and $-90 \mathrm{CAD}$. At $-270 \mathrm{CAD}$, the low speed region with a tumble structure is found to be at the center of the flow field. Since BB plane is in the middle between AA and CC planes, unlike AA and CC planes, the incoming air on $\mathrm{BB}$ plane along both intake ports partially merges into the middle plane, so the velocity magnitudes of $\mathrm{BB}$ plane are higher than that along the AA and CC planes. The stronger inertia of intake air from both intake ports is revealed using the PIV data of DD plane from an orthogonal view. At -180 and $-90 \mathrm{CAD}$, the flow behavior at $\mathrm{BB}$ plane shows a good agreement with that along $\mathrm{AA}$ and $\mathrm{CC}$ planes, which means that the in-cylinder flow has a distinct feature of tumble motion after the intake stroke. 

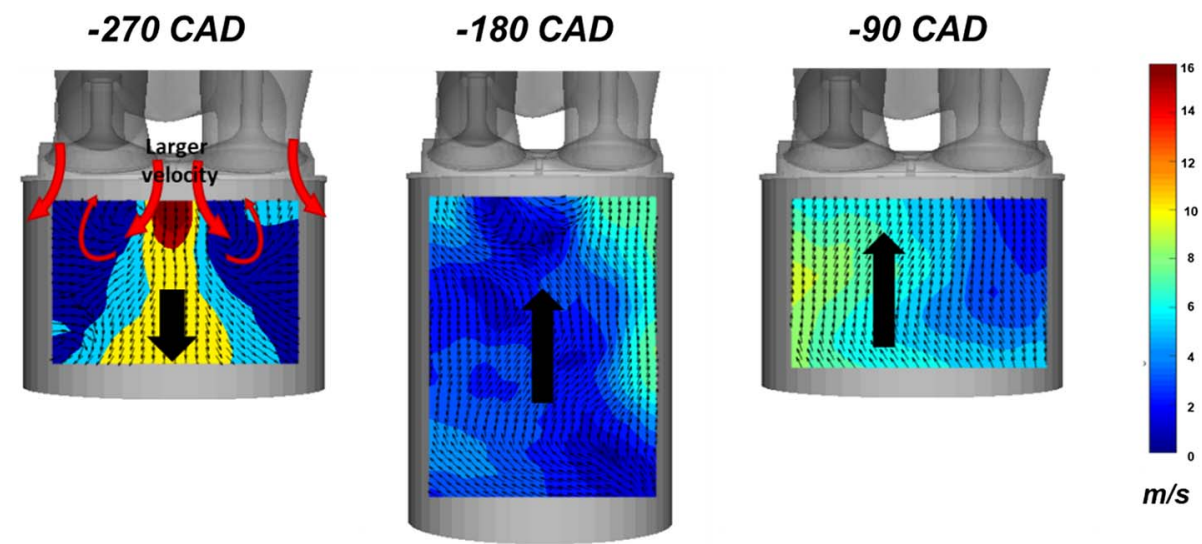

Fig. 10. PIV results at $-270,-180$, and -90 CAD of DD plane.

\subsection{PIV flow field measurements of DD and EE planes}

Planes DD and EE are perpendicular to $\mathrm{AA}, \mathrm{BB}$, and CC planes, so these two planes provide an orthogonal perspective of the flow field in the cylinder. Figure 10 shows the PIV results of DD plane. At - 270 CAD, the PIV data of DD plane shows a strong downward flow along the center region of the plane. In accordance with the results from $\mathrm{BB}$ plane, the incoming air of both two intake ports merges into the middle area between the intake valves, resulting in higher velocity magnitude.

Figure 11 shows the PIV results of BB and DD planes. The dash lines indicate the overlap part of these two planes. Both the velocity direction and magnitude show good agreement between the overlap parts of $\mathrm{BB}$ and $\mathrm{DD}$ planes. Unlike the flow between two intake valves, the flow speed and directions under the intake valves have quite different behaviors. Specifically, the velocities under the intake valves are low and pointing upwards. At -180 and -90 CADs, the flow fields of DD plane are uniform. The velocity directions are both upward. As for PIV data of plane $\mathrm{EE}$, Figure 12 shows that the main flow pattern of plane EE is similar to that along the DD plane except for the circled parts with opposite velocity directions at -270 and -180 CADs. The tumble motion, which can be clearly observed on $\mathrm{BB}$ plane, is responsible for the opposite velocity directions. Figure 11 illustrates the relative positions of $\mathrm{BB}$ plane and EE plane. The solid line shows that plane EE is sectioning through the tumble flow structure directly. From the results obtained on $\mathrm{BB}$ plane, the upper half of the tumble flow is moving upward while the bottom part is moving downward. These velocity-opposite flow features are more distinctly displayed on EE plane with an orthogonal view. These results show that the data captured from BB and EE planes are in good agreement.

\subsection{Interpretation of the in-cylinder 3-D flow structure}

The in-cylinder flow field, especially the main flow structure with larger velocity magnitude, has a strong effect on air-fuel mixing and ignition processes, which subsequently influence the combustion simulations [14]. Thus, accurate identification of flow structure with large velocity magnitude is important for reliable LES validations. Based on the flow analysis and correlations of multi-plane PIV data, Figure 13 shows the main flow structures with large velocities through a 3-D perspective. The intake valves and exhaust valves are colored as blue and orange, respectively. The main flow trends are labeled using arrows, and the colors of arrow represent the velocity magnitude. It is worth mentioning that the PIV flow fields of different planes were not recorded of the same engine cycle. To minimize the cycle-to-cycle influence, the engine operating conditions were carefully controlled during each PIV measurement. The Root Mean Square (RMS) results were also checked after completing the PIV measurement on each plane. The spatial averaged coefficient of variation (which is defined as the ratio of spatial average of ensemble RMS to the spatial average of ensemble average velocity) for each plane is of similar level with previous work [16]. Therefore, the cyclic variations of main flow trends of different planes would be considered minimal.

For LES validations, the whole flow structure obtained from multi-plane PIV measurements can provide a guidance for detailed comparisons at crucial regions. Starting at $-270 \mathrm{CAD}$, the high-speed flow regions are formed because of the intake port directions and strong incoming air. The main flows at $-270 \mathrm{CAD}$ are indicated by the arrows in Figure 13a. Two high-speed regions under the exhaust valves and between two intake valves (red arrows in Fig. 13a) should be carefully examined during validations. At $-180 \mathrm{CAD}$, the flow structure becomes tumblemotion dominant. In addition to validating the whole tumble structure, the tumble center is another crucial point to be considered. At $-90 \mathrm{CAD}$, an upward flow is dominant. The velocity direction and magnitude in the region under the intake valves should be checked carefully since the velocity magnitude is relatively large.

\section{LES validations}

After the introduction of main flow structures above, the PIV and LES results are compared in details in this section. In this study, a novel LES validation method is proposed. The flow chart depicted in Figure 14 shows the flow chart 

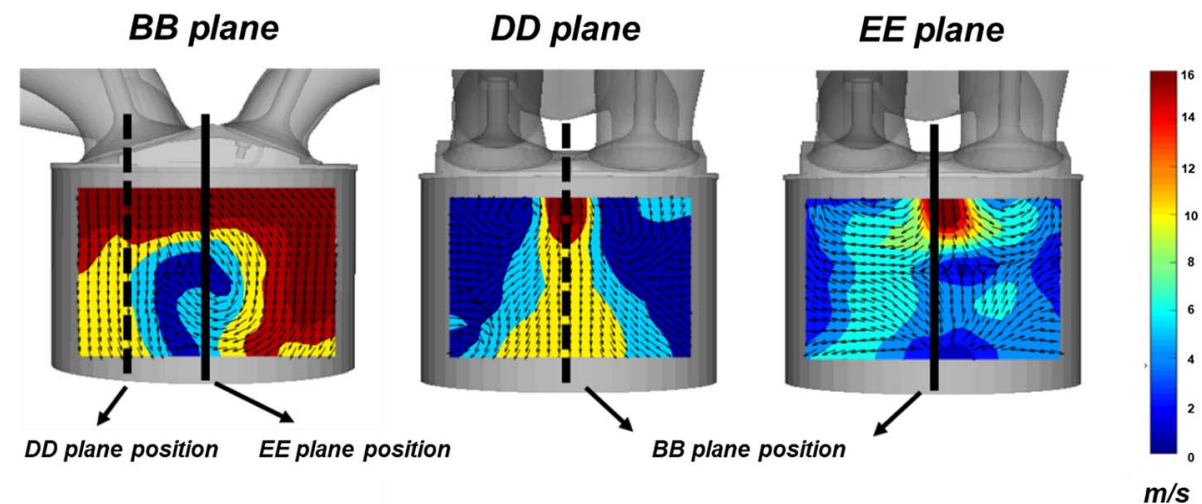

Fig. 11. PIV results at $-270 \mathrm{CAD}$ of $\mathrm{BB}, \mathrm{DD}$, and EE planes.
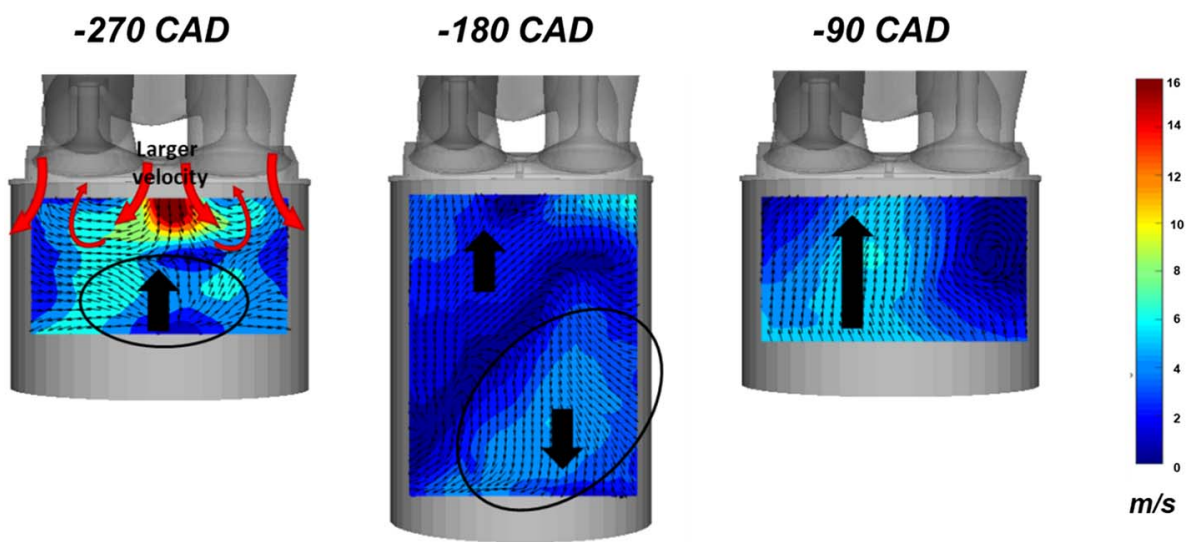

Fig. 12. PIV results at $-270,-180$, and -90 CAD of EE plane.

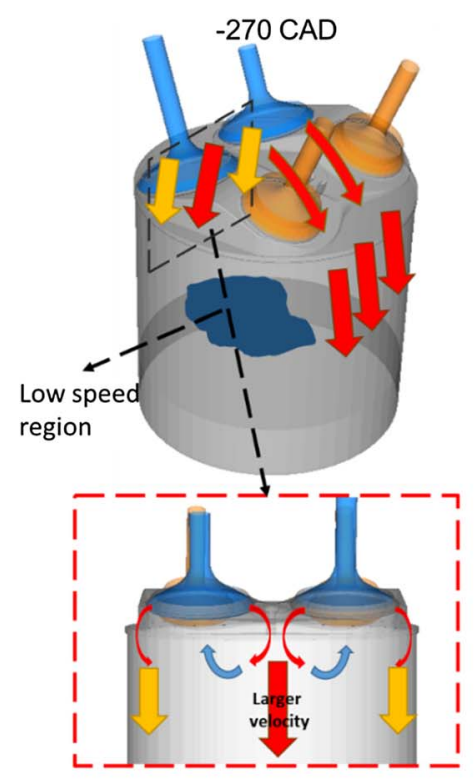

(a)

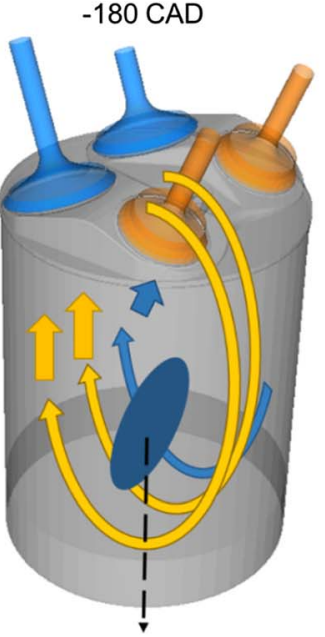

Low speed \& vortex region

(b)

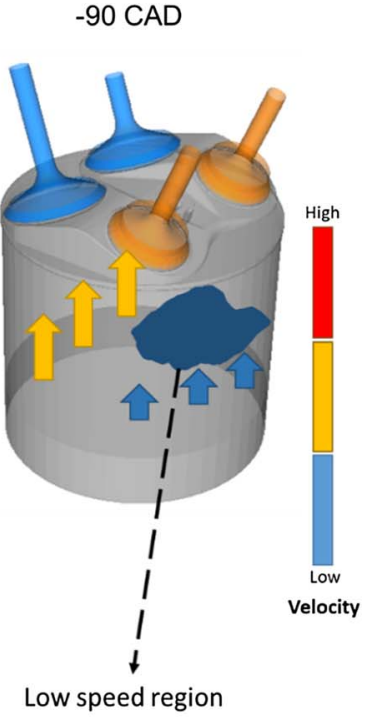

(c)

Fig. 13. Main flow structures with relatively large velocity magnitudes and low-speed regions. 


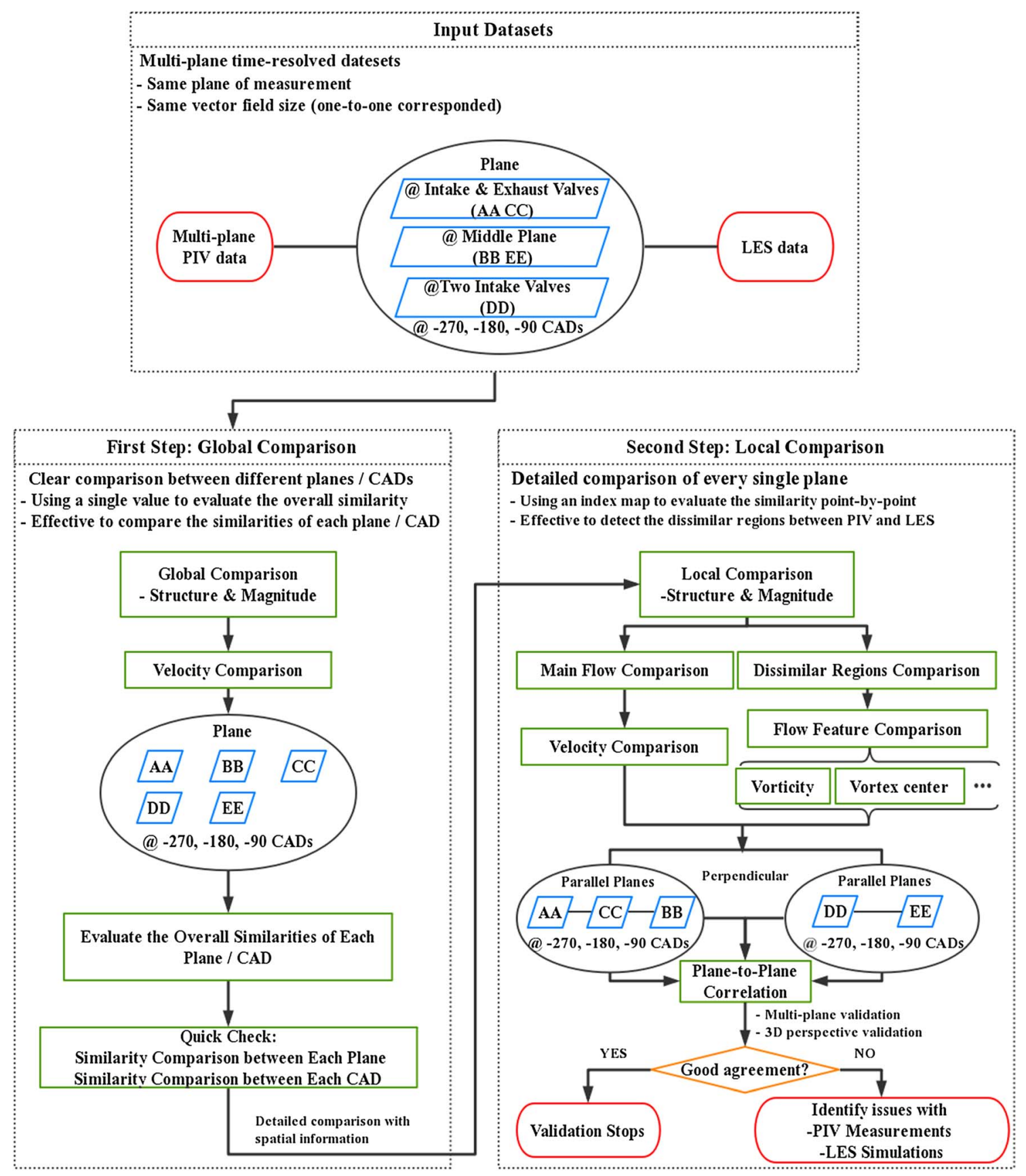

Fig. 14. Flow chart of validation process.

of the validation process. Before validations, the plane positions and the vector field sizes must be checked to be consistent between PIV and LES flow fields. The validation process can be divided into two steps: global comparison and local comparison.

The first step is known as the global comparison, which provides a quantitative single value to quickly check the overall similarities of velocity directions and magnitudes at a given CAD between PIV and LES of an individual plane. Then, the trends of similarities of different planes at various CADs can be established. While this is a relatively simple method to quantify the flow similarities, however, a single value cannot truly show the detailed comparisons with spatial information. Thus, a local point-by-point comparison method is implemented as the next step.

Unlike using a global value to compare two different data sets, local comparisons use an index map instead of a single value to evaluate the similarities between PIV and LES flow fields point-by-point. It is effective to evaluate the similarities of specific regions, so further analysis can be used to investigate the flow nature in those regions of interest, including: 
- Main flow comparison: Using multi-plane PIV measurements, the crucial regions important for engine flow development can be detected. In addition, plane-to-plane correlations can be established to check for flow similarity from a 3D perspective. If the main flow structures cannot be simulated by LES model or one of the planes shows no strong correlation with other planes, there could be potential issues with the PIV measurements or LES simulations which must be further examined carefully.

- Dissimilar regions comparison: The flow features such as vorticity strength and vortex center location could be used to identify dissimilar regions between two flow fields. The flow feature comparison in dissimilar regions is also important for the validation process. Different flow features such the tumble center location could lead to quite different flow developments. Also, planeto-plane correlations of the flow features can provide a $3 \mathrm{D}$ perspective way to validate the LES model.

\subsection{Global comparison}

The GSI and Global Magnitude Index (GMI) will be illustrated for global comparison first. The global GSI is defined as below:

$$
\mathrm{GSI}=\frac{(u \cdot v)}{\|u\|\|v\|}
$$

where $u$ and $v$ are mean velocities obtained by experiments and simulations, respectively. The GSI is used to evaluate the angle between two vectors. The range of GSI is from -1 to +1 . A value of 1 means the vectors are perfectly aligned in the same direction, while the value of -1 corresponds to two vectors with an exact opposite direction. Similarly, the GMI is defined as below:

$$
\text { GMI }=1-\left|\frac{\|u\|-\|v\| \mid}{\|u\|+\|v\|}\right|
$$

where $u$ and $v$ are of the same definitions as in GSI. However, GMI is used to evaluate the magnitude difference between two vectors. It varies from 0 to 1 . The value of 0 means a poor matching between two velocity magnitudes, while a value of 1 means that the velocity magnitude of these two vectors is identical. For both GSI and GMI, the experimental velocity $u$ and numerical velocity $v$ are phase-averaged. The definition of $\|u\|$ and $\|v\|$ are as follows:

$$
\begin{aligned}
& \|u\|=(u \cdot u)^{\frac{1}{2}}=\left(\sum_{k=1}^{n} u_{k, 1} u_{k, 1}+u_{k, 2} u_{k, 2}\right)^{\frac{1}{2}}, \\
& \|v\|=(v \cdot v)^{\frac{1}{2}}=\left(\sum_{k=1}^{n} v_{k, 1} v_{k, 1}+v_{k, 2} v_{k, 2}\right)^{\frac{1}{2}},
\end{aligned}
$$

where $u_{k}$ and $v_{k}$ are the $k$ th velocity vectors of experimental and simulated data.
Figure 15 shows the GSI and GMI results of all five planes at $-270,-180$, and -90 CADs. For global structure comparison (Fig. 15a), the similarity of the flow structure at -180 and -90 CAD (GSI $\geq 0.8$ ) is better than the case at -270 CAD (GSI values are between 0.6 and 0.8, except for $\mathrm{BB}$ plane). Because of the strong incoming air and the presence of intake valves, the transition from laminar flow to turbulence flow could happen behind the intake valves. So that it will be more challenging for LES model to accurately simulate the flow during intake stroke. As for global magnitude comparison (Fig. 15b), the velocity magnitude shows a good consistency between PIV and LES data (GMI > 0.9). Similar to the GSI results, the GMI values for compression stroke are larger than the values for intake stroke. In particular, the similarities between PIV and LES of BB plane are in good agreements in both structure (GSI $\geq 0.8$ ) and magnitude (GMI > 0.95), which is much better than the other planes.

In general, the global comparison results show that:

- Global comparison of velocity magnitude shows a better agreement than velocity direction $(\mathrm{GMI}>0.9)$.

- The flow fields during early intake stroke show less similar results between PIV and LES.

\subsection{Local comparison}

Local Structure Index (LSI) and Local Magnitude Index (LMI) maps are used to show the similarity of flow features point-by-point. The definitions of LSI and LMI are the same as GSI and GMI, respectively, as follows:

$$
\begin{gathered}
\mathrm{LSI}=\frac{\left(u_{k} \cdot v_{k}\right)}{\left\|u_{k}\right\|\left\|v_{k}\right\|}, \\
\mathrm{LMI}=1-\mid \frac{\left\|u_{k}\right\|-\left\|v_{k}\right\|}{\left\|u_{k}\right\|+\left\|v_{k}\right\|},
\end{gathered}
$$

where $u_{k}$ and $v_{k}$ are the $k$ th velocity vectors of experimental and simulated data. To show the correlations and differences between the global indices and local indices, the local comparison maps of the cases with low and high global index values are illustrated respectively.

According to the results of last section, the global index values of nearly all five planes at $-270 \mathrm{CAD}$ are lower than the other two CADs. It is because the flow is more chaotic at $-270 \mathrm{CAD}$ with strong influence by intake air and intake valve movement. Figure 16 shows the local structure and magnitude index maps of the valveinduced intake flow (plane CC at -270 CAD). Unlike using a single value to evaluate the similarity, local comparison maps can provide spatial information between PIV and LES. The dissimilar regions are indicated using dash lines. Thus, for the cases with low global index values, local comparison maps are very useful since they provide additional insights to identify the spatial distribution of dissimilar regions.

The cases with high global index values are mainly found at -180 and -90 CADs. The flow fields at these 

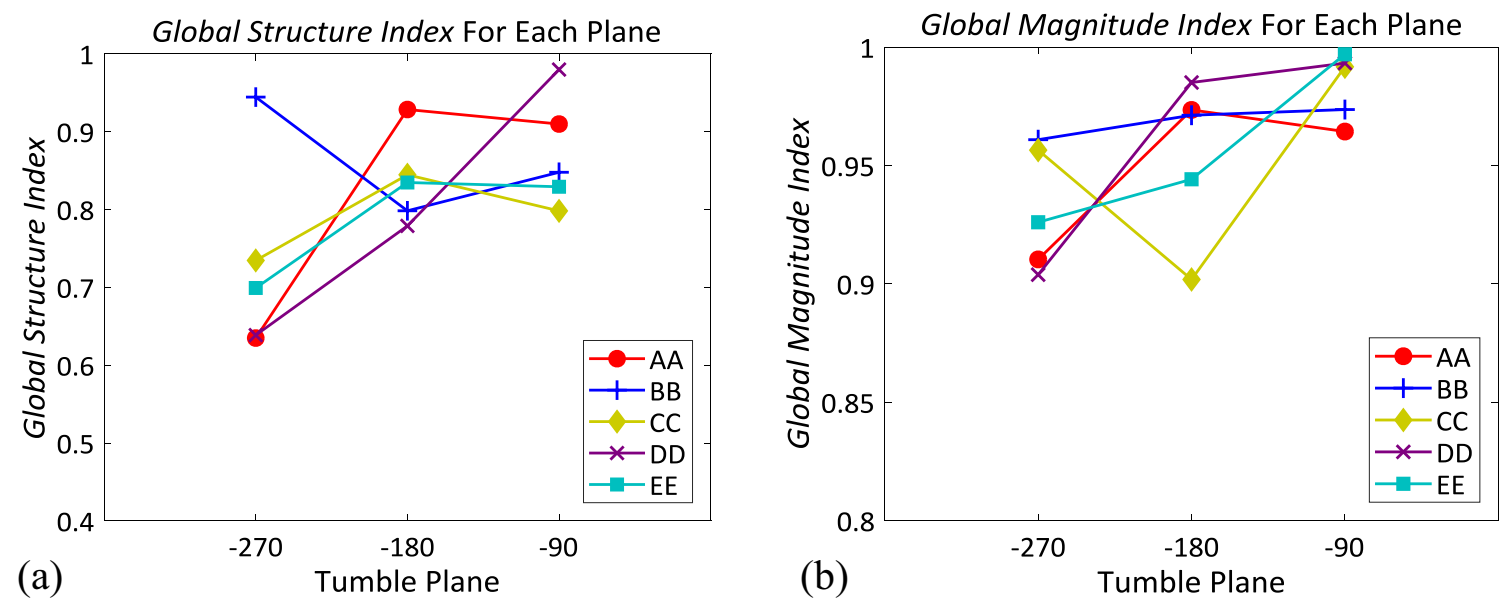

Fig. 15. GSI and GMI results of five tumble planes at $-270,-180$, and -90 CADs.
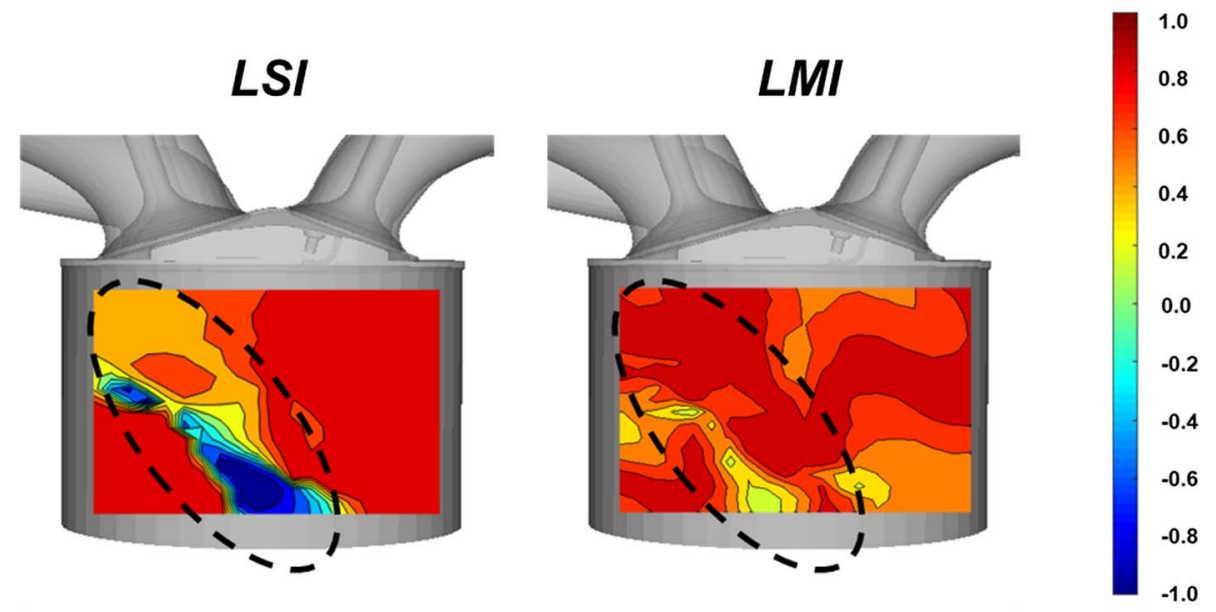

LSI / LMI

Fig. 16. Local structure and magnitude index maps of CC plane at $-270 \mathrm{CAD}$.

two CADs can be divided into two representative flow structures: tumble flow and uniform flow. Figures $17 \mathrm{a}$ and $17 \mathrm{~b}$ show the local structure and magnitude index maps of the tumble flow (plane AA at -180 CAD) and uniform flow (plane DD at -90 CAD) conditions. The global structure and magnitude indices for plane AA at -180 CAD are 0.928 and 0.974 , respectively, which reach a very high level. However, the LSI and LMI maps in Figure 17a still show distinct regions with low similarities in the middle of the tumble motion (indicated by dash lines). The results show that validation between PIV and LES using a single value is not enough. High GSI and GMI values cannot guarantee that the velocity vectors match well everywhere inside the flow fields. As for the LSI and LMI maps in Figure 17b, the results show great agreements at nearly every data point. On the regions which are dominated by uniform flow, the velocity directions obtained from PIV and LES are consistent with each other while the velocity magnitudes are slightly different.
To clarify the applicability of local comparison for valve-induced and tumble flow and global comparison for uniform flow, the global indices of smaller regions within the flow field as well as the global index of the whole flow field are compared. To show the randomness of the region selection, five smaller regions are selected to show the comparison results. One region is in the middle and the other four regions are at the corners of the flow field. Figure 18 shows a sample flow field with the locations of these five smaller regions. The size of the smaller region is 6 by 6 vectors. The GSI values of these smaller regions within different flow fields (valve-induced flow, tumble flow and uniform flow) and the standard deviations are shown in Table 3.

According to Table 3, the standard deviations of GSI values for valve-induced flow and tumble flow are much larger than uniform flow case. In addition, the GSI values of the whole flow field are 0.928 and 0.735 for valve-induced flow and tumble flow, respectively, which are quite different 
LSI

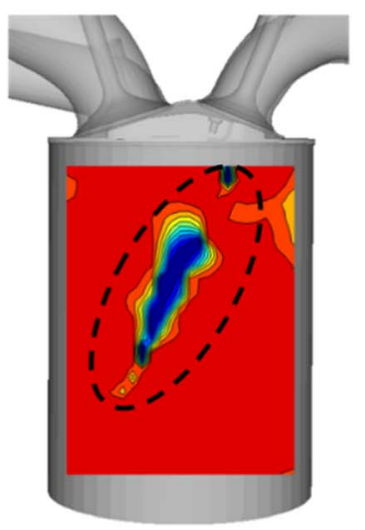

LMI

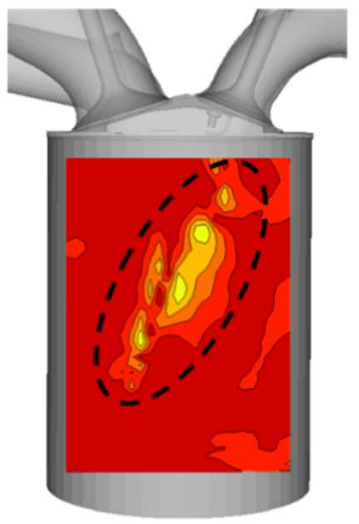

LSI

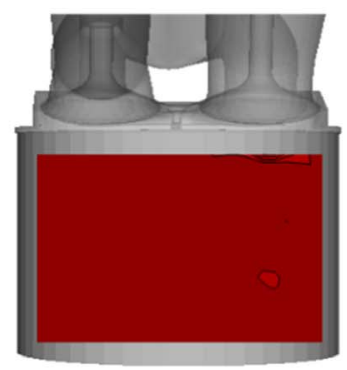

LMI

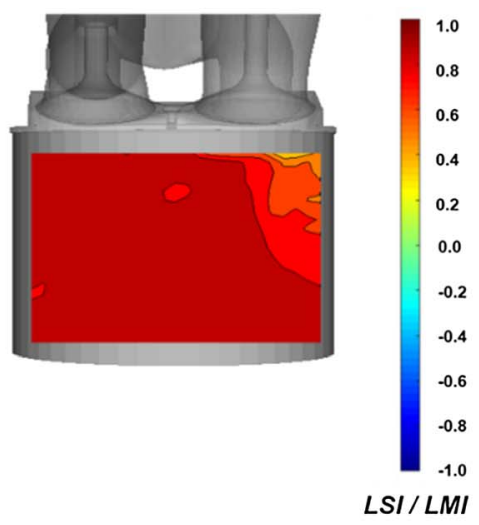

(a)

(b)

Fig. 17. LSI and LMI maps of (a) plane AA at -180 CAD; and (b) plane DD at -90 CAD.

$6 \times 6$

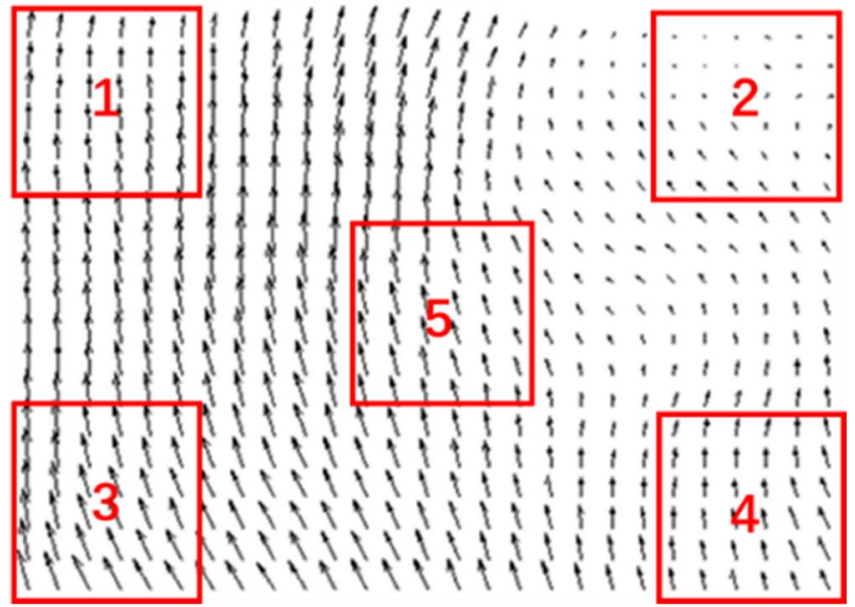

Fig. 18. Locations of five smaller regions inside the flow field.

with some of the GSI values (in red) of these five regions. However, the standard deviation is small and the GSI values of all five regions are similar with GSI value (0.980) of the whole flow field for uniform flow case.

The above results show the needs of applying local comparison maps to evaluate the comparison of flow data except for the uniform flow cases, even though the global indices show good agreements. For uniform flow field, the global comparison is generally adequate to evaluate the similarity between PIV and LES results.

\subsection{Main flow comparison}

Based on the main flow structures of the multi-plane PIV experiments, three representative flow structures can be found, which are valve-induced intake flow, tumble flow, and uniform flow. Since the global indices are good indicators for the similarities of uniform flow, the valve-induced intake flow and tumble flow are investigated using local comparison maps in this section.

\subsubsection{Valve-induced intake flow (at - 270 CAD)}

During intake stroke, the in-cylinder flow is mainly influenced by the incoming air and intake valve movements. The flow data of $\mathrm{BB}$ plane (between two intake valves) and $\mathrm{CC}$ plane (right under the intake valve and exhaust valve) are selected to illustrate the local structure and magnitude comparisons of main flow. Figure 19 shows the local flow comparison results of BB plane, including the PIV (main flow pattern is indicated using black arrows) and LES results and LSI and LMI maps. From previous section of global comparison, the GSI and GMI values of BB plane show a much better matching than CC plane at -270 CAD. The reason is that the effects of stronger incoming air from both intake ports and less influence by the movements of intake valves make the flow at BB plane not as disordered as that found on $\mathrm{CC}$ plane. As expected, the LSI and LMI maps also show higher consistency between PIV and LES results on BB plane compared with the LSI and LMI results on CC plane. For the main flow, both the structure and magnitude show high similarities between PIV and LES flow fields. The only dissimilar region detected by both $\mathrm{LSI}$ and LMI maps is at the middle of the measured plane where tumble motion exists.

For the flow comparison at the location right under the intake valve and exhaust valve, Figure 20 shows the PIV results of main flow pattern, LES results, and LSI and LMI maps at -270 CAD of CC plane. Both the LSI and LMI results show that the dissimilar regions are under the intake valves (within the dash lines). As for the main flows indicated by the black arrows, local comparison values show good agreement even though the global comparison values are low.

The local comparison results of BB and CC planes show the same trend as GSI values with a better agreement along the middle plane. Moreover, the local comparison map can 
Table 3. Global structure indices of zones $1-5$.

\begin{tabular}{lcccccc}
\hline Global Structure Index (GSI) & Zone 1 & Zone 2 & Zone 3 & Zone 4 & Zone 5 & SD \\
\hline Valve-induced flow (plane CC @ -270 CAD) & 0.502 & 0.959 & 0.930 & 0.988 & 0.554 & 0.237 \\
Tumble flow (plane AA @ -180 CAD) & 0.856 & 0.858 & 0.995 & 0.903 & 0.242 & 0.301 \\
Uniform flow (plane DD @ -90 CAD) & 0.993 & 0.960 & 0.998 & 0.996 & 0.990 & 0.016 \\
\hline
\end{tabular}

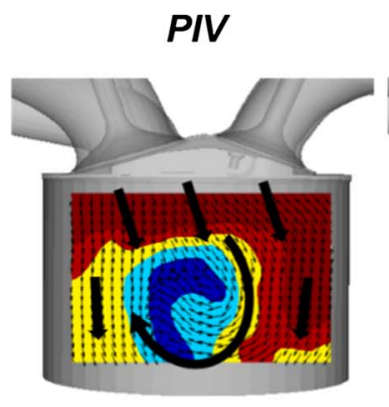

(a)

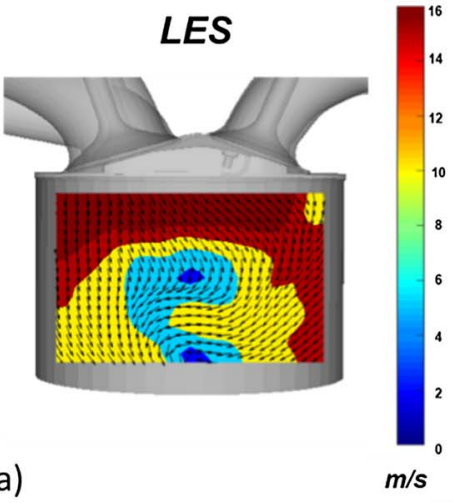

$m / s$

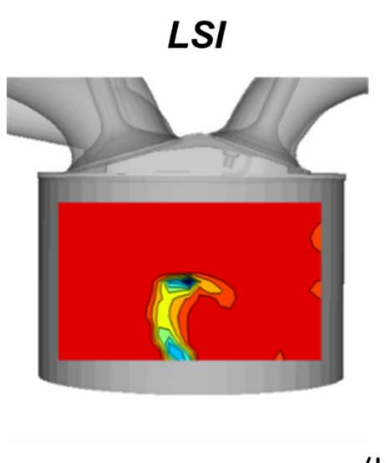

(b)

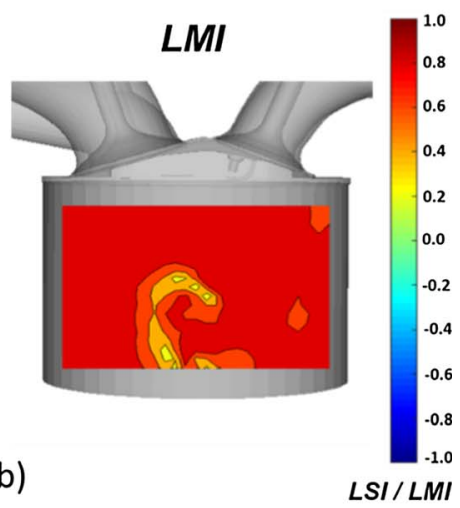

$L S I / L M I$

Fig. 19. (a) PIV (with main flow pattern) and LES results of BB plane at -270 CAD; (b) LSI and LMI maps of BB plane at $-270 \mathrm{CAD}$

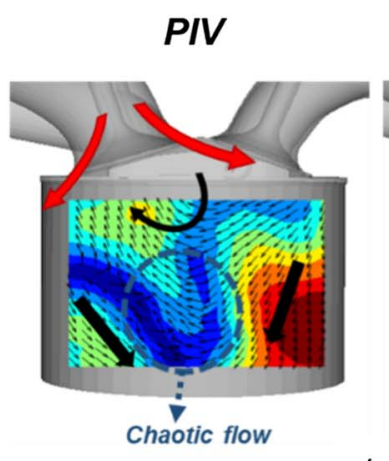

(a)

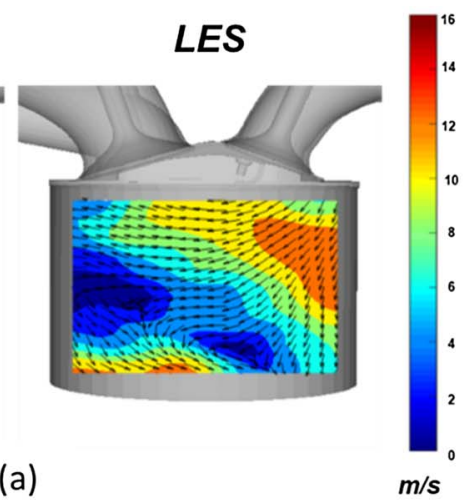

$\mathrm{m} / \mathrm{s}$

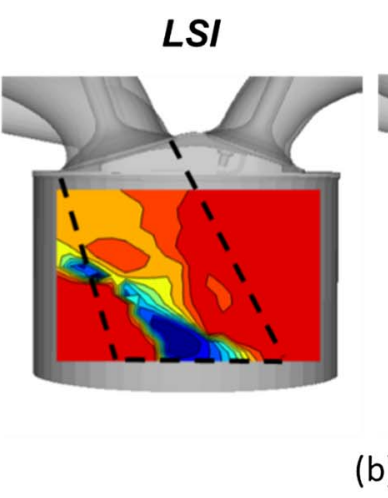

(b)

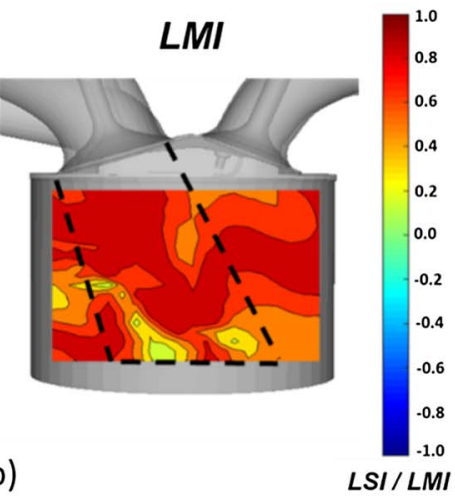

Fig. 20. (a) PIV (with main flow pattern) and LES results of CC plane at -270 CAD; (b) LSI and LMI maps of CC plane at $-270 \mathrm{CAD}$.

also identify the dissimilar regions between PIV and LES. The good matching of the main flow structure and magnitude on CC plane demonstrate that low global index value cannot represent the similarity of the main flow pattern.

\subsubsection{Tumble flow (at - 180 CAD)}

With the closing of intake valves, the tumble flow structures are gradually formed at $-180 \mathrm{CAD}$. The flow regions change from near valve area to the whole flow domain inside the cylinder. For this large-scale flow structure, the plane-to-plane correlations provide an improved 3D understanding of the flow, so the flow data of three parallel planes
$(\mathrm{AA}, \mathrm{BB}$, and $\mathrm{CC})$ and their perpendicular plane $(\mathrm{EE})$ is discussed in this part. The main flow structures of AA, $\mathrm{BB}$, and CC planes show strong tumble motions at -180 CAD. Figure 21 shows the PIV results of the main flow pattern, LES results, and LSI and LMI maps at $-180 \mathrm{CAD}$ of AA, BB, and CC planes. It can be seen that the dissimilar regions are mainly located in the middle of the tumble motion. For the main flow regions with larger velocities, the PIV and LES results show good similarity with strong tumble clockwise direction as the main flow structures. Regarding the tumble locations and directions, the flow fields of these three planes seem to be very well correlated. 


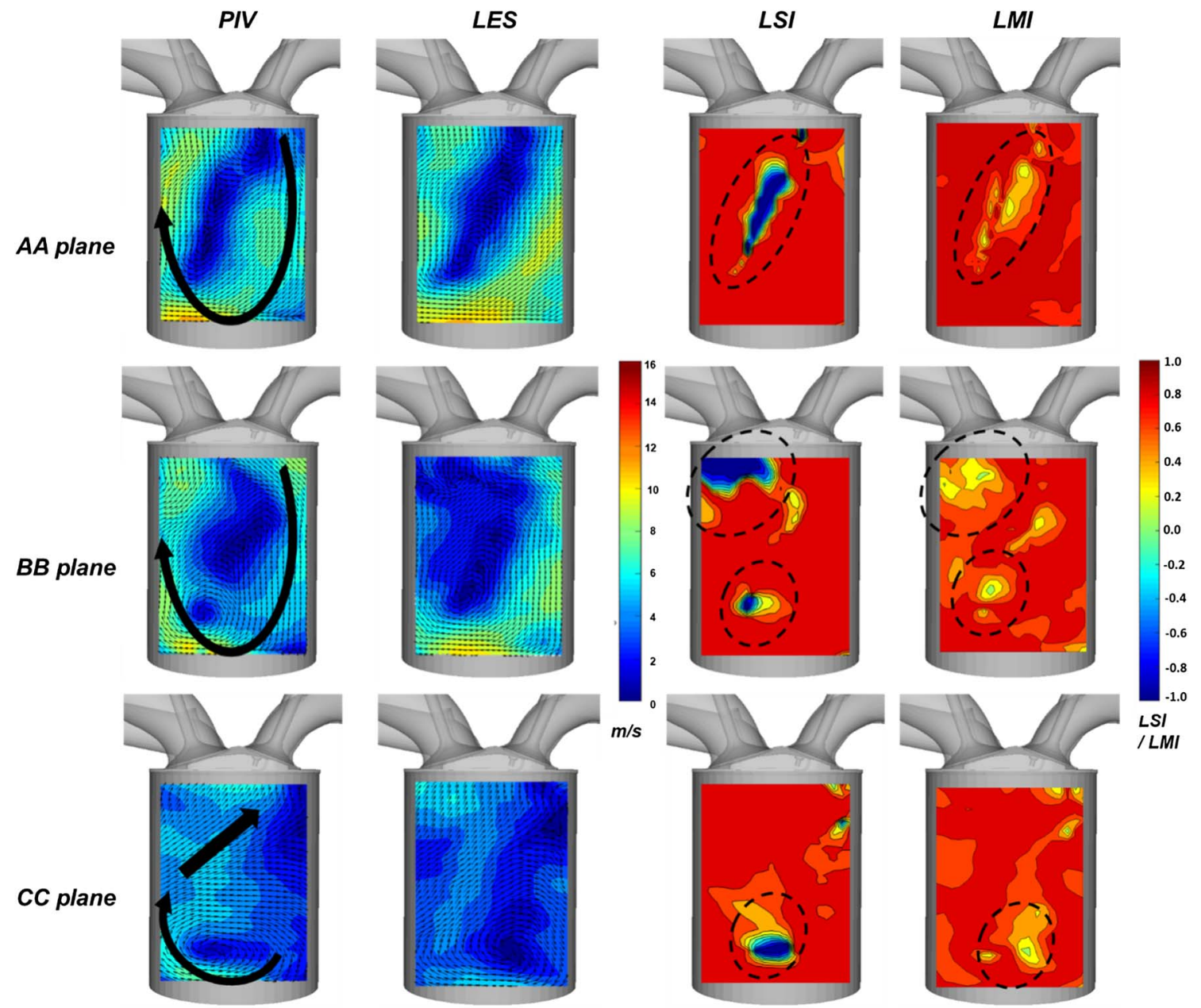

Fig. 21. PIV (with main flow pattern) and LES results and LSI and LMI maps of AA, BB, and CC planes at -180 CAD.
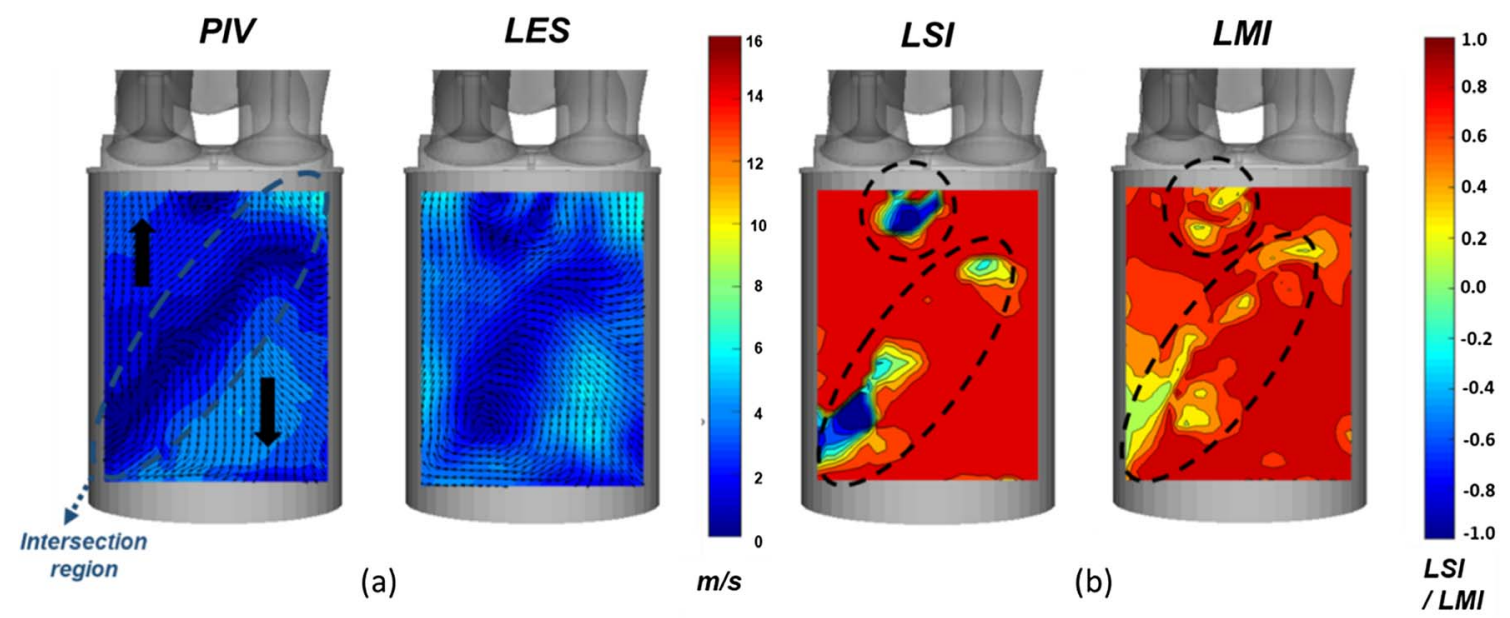

Fig. 22. (a) PIV (with main flow pattern) and LES results of EE plane at -180 CAD; (b) LSI and LMI maps of EE plane at -180 CAD.

Figure 22 depicts the PIV and LES results and LSI and LMI maps of EE plane, which show an orthotropic view of $\mathrm{AA}, \mathrm{BB}$, and CC planes. The main flow comparison of PIV and LES results shows an overall good agreement while the differences are observed at the intersecting zones (circled in Fig. 22). Although the velocity profiles are not comparable 
inside the intersection zones, the locations of these two intersecting zones are quite similar. For the same reason as discussed before (Fig. 11), the intersection zone exists because plane EE is directly cutting through the tumble motion, and the intersection zone indicates the tumble motion locations on planes AA, BB, and CC. The similarity of the locations of intersection zones shows the tumble motion locations of $\mathrm{AA}, \mathrm{BB}$, and $\mathrm{CC}$ planes of PIV and LES results are similar as well.

At -180 CAD, the LSI and LMI maps identify the dissimilar regions which are mainly located in the middle of the tumble motion. The main flow and plane-to-plane correlations exhibit good agreements in both structure and magnitude with PIV and LES. In addition, the dissimilar regions shown on the LSI and LMI maps are found to be at the same locations. Building upon the information of the locations of dissimilar regions, detailed analysis of the flow features can be conducted to identify the issues of the mismatch.

\subsection{Dissimilar regions comparison}

Although LES model can simulate the flow in crucial regions with accurate structures and velocity magnitudes, some dissimilar regions between PIV and LES still exist. In this part, the flow feature comparison is conducted to check the relevance of the flow in dissimilar regions. According to the results of local comparison, the flow in the dissimilar regions can be divided into two types. The first type is the region with complex flow features at $-270 \mathrm{CAD}$ (Fig. 23a), which can be represented by the flow under the intake valves during intake stoke. The second type is the flow in the middle of the complete tumble motion at -180 CAD (Fig. 23b).

To analyse the flow features of these two types of flows, the velocity directions and magnitudes are two critical points to check as before. For velocity magnitude, the common feature easily found is that the dissimilar regions are of low-speed flow. For velocity direction analysis, the streamlines of PIV and LES results are shown in Figure 23. A vortex motion is defined as the flow motion leading to circular or nearly circular streamlines. From Figure 23, both the streamlines of PIV and LES show vortex-like features, and therefore the vorticity maps and Vortex Center Index (VCI) can be used to evaluate the vortex features of these curved streamlines [21].

Vorticity represents the strength of rotation at a specific point. In other words, it shows the tendency of rotating motion of a continuum at such specific point. For 2D flow fields with a flow field $\vec{V}=\left(v_{x}, v_{y}, 0\right)$, the vorticity $\vec{\omega}$ can be defined as follows:

$$
\begin{aligned}
\vec{\omega}=\nabla \times \vec{V} & =\left(\frac{\partial}{\partial x}, \frac{\partial}{\partial y}, \frac{\partial}{\partial z}\right) \times\left(v_{x}, v_{y}, 0\right) \\
& =\left(\frac{\partial v_{y}}{\partial x}-\frac{\partial v_{x}}{\partial y}\right) \hat{k},
\end{aligned}
$$

where $\hat{i}, \hat{j}$, and $\hat{k}$ are unit vectors along $x-, y$, and $z$ - axis, respectively. The vorticity is a vector, and the direction of $\vec{\omega}$ is along $z$-axis for $2 \mathrm{D}$ flow fields. The unit

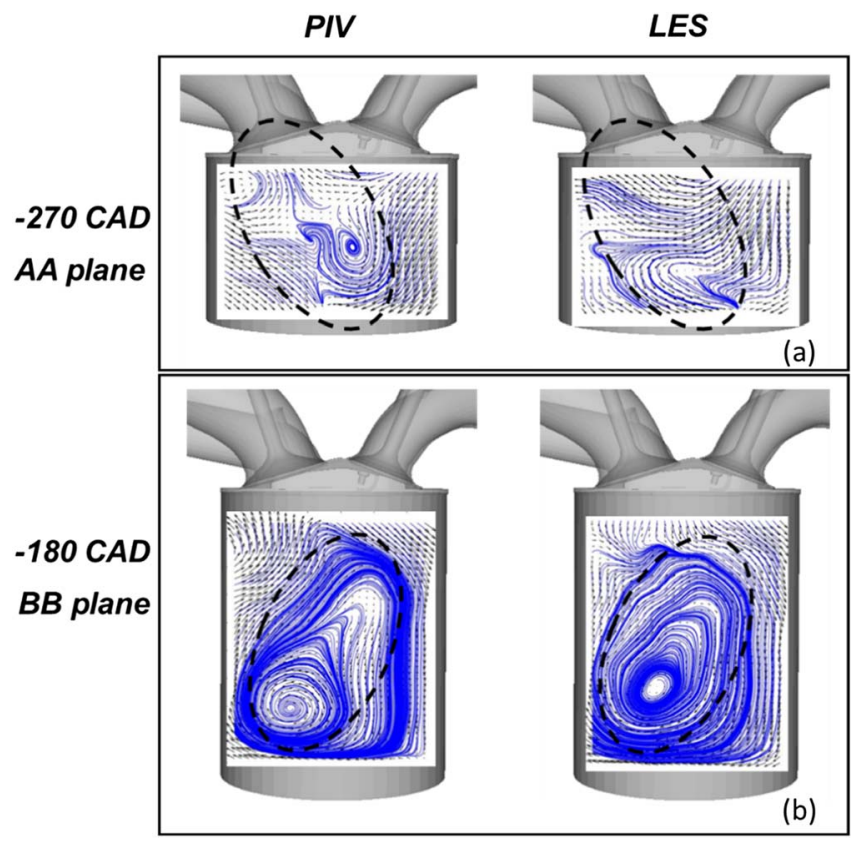

Fig. 23. Two types of dissimilar regions. (a) Chaotic flow behind the intake valves; (b) flow in the middle of a tumble motion.

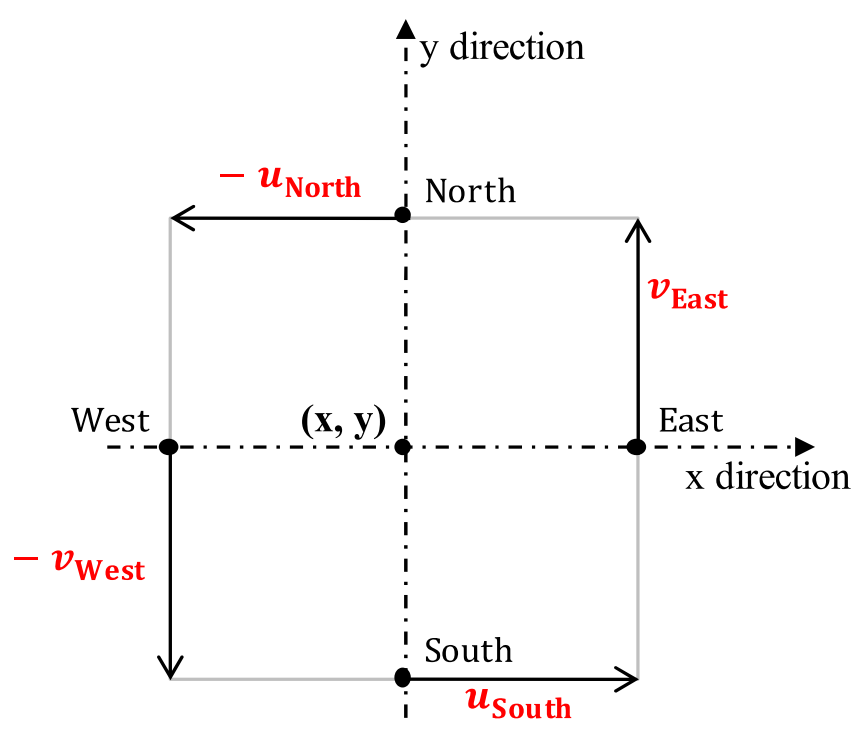

Fig. 24. Example vector field with VCI of +4 .

of vorticity is $\mathrm{s}^{-1}$. Negative vorticity values mean that the continuum will rotate in clockwise direction. Positive vorticity values mean that motion is counter-clockwise.

VCI is used to identify the vortex center locations. It is calculated by the directions of the four neighbouring normalized velocity vectors. Figure 24 shows an example with a $\mathrm{VCI}$ value of +4 . The equation of calculating $\mathrm{VCI}$ value is below:

$$
\mathrm{VCI}_{(x, y)}=v_{\text {East }}-u_{\text {North }}-v_{\text {West }}+u_{\text {South }} .
$$




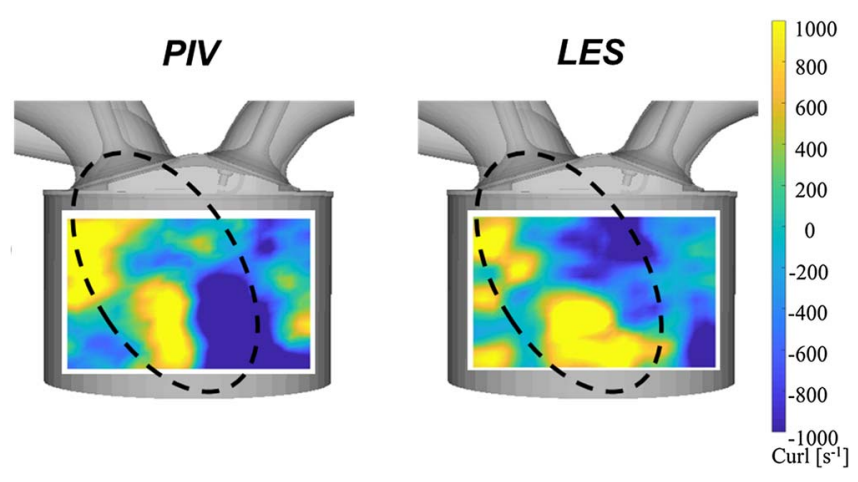

Fig. 25. Vorticity maps of dissimilar regions at $-270 \mathrm{CAD}$ of AA plane.

The range of VCI value is from -4 to +4 . The VCI values will be calculated for every data point in the flow field, and the vortex center is located at the data point with maximum absolute VCI value [21]. VCI only concentrates on the velocity directions while the vorticity maps can be calculated to evaluate the strength of the rotation. Applying both VCI and vorticity maps can avoid finding the irrotational vortex, which is a flow structure only with vortex structure without any vorticity strength.

\subsubsection{Dissimilar regions under intake valves}

For the first type of dissimilar regions, only vorticity map is used since there is no clear vortex motion. Figure 25 shows the vorticity maps of dissimilar regions under the intake valves. Unlike the poor matching of velocities, the vorticity comparison shows some reasonable correlations. The flow on the left side of the dissimilar region shows a counterclockwise motion, while the flow on the right side has a clockwise motion. The vorticity maps show the dissimilarity of the velocity is caused by the interaction of two flows with opposite vortex features. Inside the intersecting area, the velocity magnitudes are small, and the complex variations of velocity directions usually exist. As a result, the mismatch of the velocities is usually unavoidable. For these regions, identifying the flow feature can be an alternative criterion to evaluate the flow similarity.

\subsubsection{Dissimilar regions with a complete vortex}

At $-180 \mathrm{CAD}$, a dominant tumble motion with clockwise direction is found using the main flow comparison. Figure 26 shows the vorticity maps of dissimilar regions at $-180 \mathrm{CAD}$ of BB plane. The tumble motion at $-180 \mathrm{CAD}$ is clockwise so that both the vorticity maps show negative values.

For the dissimilar regions with a complete vortex motion, the location of the vortex center is an important vortex feature to examine. For a large-scale tumble motion, different vortex center locations could lead to different flow developments. Thus, the vortex center comparison is another important criterion for the validation of the flow with tumble motions as well as the main flow pattern comparison. Similar with the main flow comparison at
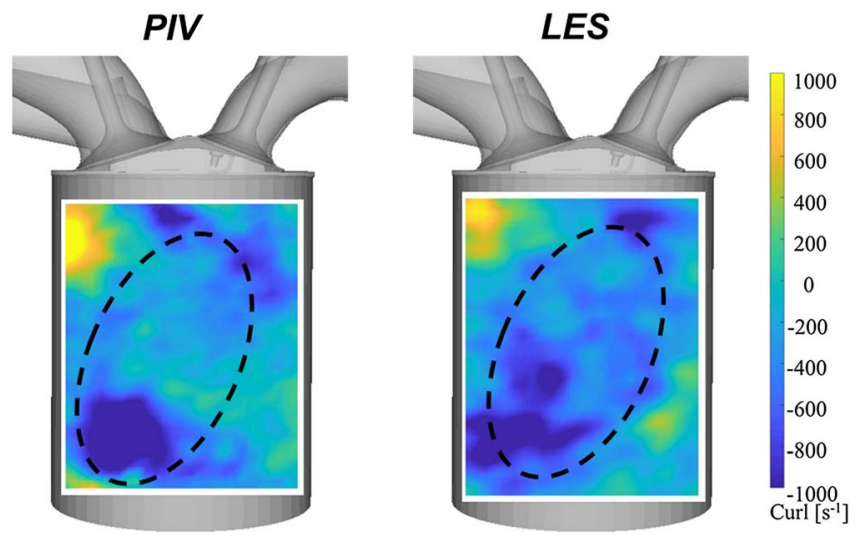

Fig. 26. Vorticity maps of dissimilar regions at $-180 \mathrm{CAD}$ of BB plane.
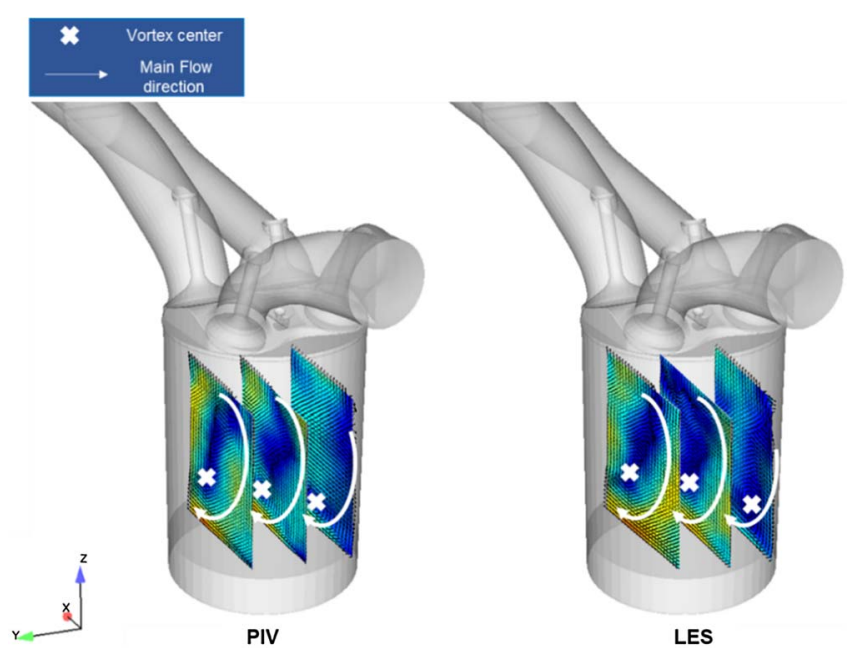

Fig. 27. Vortex center locations and directions at $-180 \mathrm{CAD}$ of $\mathrm{AA}, \mathrm{BB}$, and $\mathrm{CC}$ planes.

-180 CAD, the plane-to-plane correlations should also be considered in addition to single-plane comparison. To clearly show the plane-to-plane correlations, Figure 27 shows the vortex center locations and directions identified by VCI at $-180 \mathrm{CAD}$ of $\mathrm{AA}, \mathrm{BB}$, and $\mathrm{CC}$ planes from a $3 \mathrm{D}$ perspective. The vortex centers and the main flow directions are indicated using the cross marks and arrows, respectively. Both the main flow patterns and the vortex center locations of each plane show very good agreements. In addition, the plane-to-plane correlations are strong among these three planes for both PIV and LES results. If we treat the vortex motion from a $3 \mathrm{D}$ perspective, it can be described as a "vortex tube". It has length and direction information in $Y$-axis direction (Fig. 27). The check of the "vortex tube" locations and length can improve the accuracy of the validation. Compared with single-plane comparison, the plane-to-plane correlation of multi-plane comparison can provide insights of 3D tumble structure, and it can be helpful to validate the LES model. 


\section{Conclusion}

In this paper, multi-plane time-resolved PIV measurements are implemented to give a panoramic perspective of in-cylinder flow characteristics. A two-step process to validate the LES model has been presented. First, the global indices including GSI and GMI are formulated to directly evaluate the structure and magnitude similarities of PIV and LES flow fields and identify the specific conditions to be focused on. Then, the local "point-by-point" comparison is applied to quantitatively determine the spatial variations and dissimilarities of flow features between the PIV and LES data sets. The main findings and contributions of this work are summarized as follows:

1. Multi-plane PIV measurements improve the panoramic elucidation of the in-cylinder flow structures, allowing more comparisons of flow field along different planes between PIV and LES datasets. Multi-plane (using both parallel and perpendicular planes) comparison is more rigorous and convincing since it can also provide the information of plane-to-plane correlations in additional to typical single-plane comparison.

2. A novel validation method combining global and local comparisons is presented to validate the LES model in this study. Global comparison uses a single value to evaluate the overall similarity between PIV and LES results, which can show a clear comparison between different planes at different CADs. However, the detailed comparison with spatial information cannot be revealed by a single value alone. Therefore, local comparison is used to compare the data of every single plane in details by using the point-by-point similarity to create the velocity magnitude and structure index maps. These maps are effective to identify the dissimilar regions between PIV and LES results. Not only can the method of combining global and local comparisons show the trends of similarities at different planes and CADs, it also detects the dissimilar regions as well.

3. According to the local comparison results, the regions with complex flow structures and low-speed flows are likely to show low similarities of the velocity fields. When such situation exists, flow feature comparison instead of comparing velocity vectors directly could be taken as an alternative way to evaluate the similarity of the flows in dissimilar regions and identify possible issues with PIV measurements and LES simulations. For instance, the meshing resolution of LES model has crucial impacts on simulating accuracy and computational time. Using the validation process in this paper, the regions where dissimilarity is frequently occurred or nearly not occurred can be clearly identified. The grid size can be adjusted based on the comparison results to optimize the simulating accuracy and speed.

Future work needs to focus on comparing the PIV and LES results at a higher CAD resolution. The crank angleresolved analysis can evaluate the comparison from early intake stroke to the late compression stroke for more transient and cycle-to-cycle comparison of in-cylinder flow validation.

Acknowledgments. This research is sponsored by the General Motors Company (USA). It was carried out at the National Engineering Laboratory for Automotive Electronic Control Technology of Shanghai Jiao Tong University. The technical discussions provided by Dr. Tang-Wei Kuo (retired from General Motors Global R\&D) are gratefully acknowledged.

\section{References}

1 Rutland C.J. (2011) Large-eddy simulations for internal combustion engines - A review, Int. J. Engine Res. 12, 4, 421-451.

2 Enaux B., Granet V., Vermorel O., Lacour C., Pera C., Angelberger C., Poinsot T. (2011) LES study of cycle-tocycle variations in a spark ignition engine, Proc. Comb. Inst. 33, 2, 3115-3122.

3 Van Dam N., Sjöberg M., Som S. (2018) Large-eddy simulations of spray variability effects on flow variability in a direct-injection spark-ignition engine under non-combusting operating conditions, SAE International, SAE Technical Paper No. 2018-01-0196.

4 Kuo T.-W., Yang X., Gopalakrishnan V., Chen Z. (2014) Large-Eddy Simulation (LES) for IC engine flows, Oil Gas Sci. Technol. - Rev. IFP Energies nouvelles 69, 1, 61-81.

5 Yang X., Kuo T.-W. (2017) Correlation of CCV between incylinder swirl ratio and polar velocity profile in valve seat region using LES under motored engine condition, Oil Gas Sci. Technol. - Rev. IFP Energies nouvelles 72, 38.

6 Sick V., Reuss D., Rutland C., Haworth D., Oefelein J., Janicka J., Kuo T.-W., Yang X., Freitag M. (2010) A common engine platform for engine LES development and validation, LES4ICE Conference, 18-19 Nov, Rueil-Malmaison, France, .

7 David L.R., Zhong Z., Yang X., Kuo T.-W., Sick V. (2018) Measured and LES motored-flow kinetic energy evolution in the TCC-III engine, SAE International, SAE Technical Paper No. 2018-01-0192.

8 Nicollet F., Krüger C., Schorr J., Nicoud E., Colin O., Angelberger C., Bode J., Böhm B. (2017) A PIV-guided large-eddy simulation of in-cylinder flows, Oil Gas Sci. Technol. - Rev. IFP Energies nouvelles 72, 28.

9 Chang Y., Wu A., Reuss D., Sick V. (2018) Scale similarity analysis of internal combustion engine flows - Particle image velocimetry and large-eddy simulations, SAE International, SAE Technical Paper No. 2018-01-0172.

10 Buhl S., Hartmann F., Kaiser S.A., Hasse C. (2017) Investigation of an IC engine intake flow based on highly resolved LES and PIV, Oil Gas Sci. Technol. - Rev. IFP Energies nouvelles $\mathbf{7 2}, 15$.

11 Abraham P., Liu K., Haworth D., Reuss D., Sick V. (2014) Evaluating Large-Eddy Simulation (LES) and high-speed Particle Image Velocimetry (PIV) with phase-invariant Proper Orthogonal Decomposition (POD), Oil Gas Sci. Technol. - Rev. IFP Energies nouvelles 69, 1, 41-59.

12 Buhl S., Gleiss F., Köhler M., Hartmann F., Messig D., Brücker C., Hasse C. (2017) A combined numerical and experimental study of the 3D tumble structure and piston 
boundary layer development during the intake stroke of a gasoline engine, Flow Turbul. Combust. 98, 2, 579-600.

13 Janas P., Wlokas I., Bohm B., Kempf A. (2017) On the evolution of the flow field in a spark ignition engine, Flow Turbul. Combust. 98, 237-264.

14 Bode J., Schorr J., Krüger C., Dreizler A., Böhm B. (2016) Influence of three-dimensional in-cylinder flows on cycle-tocycle variations in a fired stratified DISI engine measured by time-resolved dual-plane PIV, Proc. Combus. Inst. 36, 3, 3477-3485.

15 Baum E., Peterson B., Surmann C., Michaelis D., Böhm B., Dreizler A. (2013) Investigation of the 3D flow field in an IC engine using tomographic PIV, Proc. Combus. Inst. 34, 2, 2903-2910.

16 Schiffmann P., Gupta S., Reuss D., Sick V., Yang X., Kuo T.-W. (2016) TCC-III engine benchmark for large-eddy simulation of IC engine flows, Oil Gas Sci. Technol. - Rev. IFP Energies nouvelles 71, 3 .
17 Bücker I., Karhoff D.C., Klaas M., Schröder W. (2012) Stereoscopic multi-planar PIV measurements of in-cylinder tumbling flow, Exp. Fluids 53, 6, 1993-2009.

18 Werner H., Wengle H. (1993) Large-eddy simulation of turbulent flow over and around a cube in a plate channel, Turbul. Shear Flows 8, 155-168.

19 Senecal P.K., Richards K.J., Pomraning E., Yang T., Dai M.Z., McDavid R.M., Patterson M.A., Hou S., Shethaji T. (2007) A new parallel cut-cell cartesian CFD code for rapid grid generation applied to in-cylinder diesel engine simulations. SAE Technical Paper No. 2007-01-0159.

20 Pomraning E., Rutland C.J. (2002) A dynamic one-equation non-viscosity LES model, AIAA J. 40, 4, 689-701.

21 Zhao F., Ge P., Zhuang H., David L.S. (2017) Analysis of crank angle-resolved vortex characteristics under high swirl condition in a spark-ignition direct-injection engine, J. Eng. Gas Turb. Power 140, 9. 